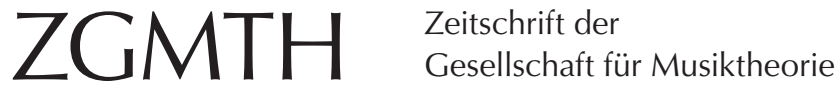

Neufeld, Marc (2017): Eine Systematik diatonischer Skalen. ZGMTH 14/2, 315-359.

https://doi.org/10.31751/939

(C) 2017 Marc Neufeld (marc.neufeld@web.de)

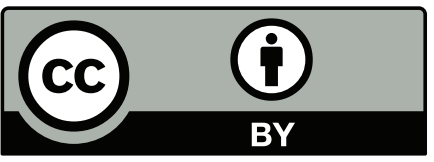

Dieser Text erscheint im Open Access und ist lizenziert unter einer Creative Commons Namensnennung 4.0 International Lizenz.

This is an open access article licensed under a Creative Commons Attribution 4.0 International License.

eingereicht / submitted: 28/01/2016

angenommen / accepted: 29/04/2016

veröffentlicht / first published: 31/12/2017

zuletzt geändert / last updated: 13/09/2018 


\title{
Eine Systematik diatonischer Skalen ${ }^{1}$
}

\author{
Marc Neufeld
}

ABSTRACT: Nach einleitenden Begriffsklärungen wird ausgehend von der Heptatonik und den Alterationsmöglichkeiten der Pedalharfe sowie von halbtönig-gleichstufigen Notationskonventionen eine Möglichkeit zur Systematisierung diatonischer Skalen vorgestellt. Die Systematik beruht auf dem Verfahren der Alteration von Stammtönen. So entsteht durch sukzessives Wegstreichen der sieben b-Vorzeichen der Ces-Dur-Skala die Kategorie der ssubtraktiven Skalen', aus diesen durch die erneute Alteration mit \#-Vorzeichen die der sadditiven Skalen . Die dritte und vierte Kategorie bilden reduzierte، Skalen (Skalen mit weniger als sieben Tönen) und serweiterte`Skalen (Skalen mit hinzugefügten Tönen, nachdem andere weggestrichen wurden). Die Systematik enthält neben traditionell verortbaren Skalen (z.B. ’Kirchentonarten`, ‘Blues-Tonleitern`) auch viele bislang nicht benannte und systematisierte Skalen. Die Kriterien der Systematik werden reflektiert und begründet, ebenso Systematisierungsentscheidungen, die durch Phänomene im Grenzbereich zwischen den definierten Skalenklassen notwendig werden. Die Grenzen der durch die Systematik eröffneten Möglichkeiten werden diskutiert. Abschließend wird der Begriff »new modality« zur Bezeichnung einer Verbindung von skalarem und spektralem Denken aufgegriffen.

After preliminary definitions of concepts and terminology, a possibility for systematizing diatonic scales is presented, building on the heptatonic system and the possibilities of alteration on the pedal harp as well as notation conventions geared to semitonally based equal temperament. This system is based on the alteration of natural notes. By gradually deleting the seven b-accidentals of the $\mathrm{C} b$ major scale, the category of 'subtractive scales' is developed, which may, by addition of \#-accidentals, be transformed into 'additive scales'. The third and fourth categories are 'reduced scales' (consisting of less than seven tones) and 'extended scales' (scales with tones added after deleting others). The system contains, besides traditional scales (e.g. medieval and renaissance modes, Blues scales), many others that have not been systematized so far. The article thinks through and legitimates the criteria of the systematization as well as decisions following from ambiguous phenomena in the threshold range between defined classes of scales. The limits of the system's possibilities are discussed. Finally, the article considers the concept of "new modality" which refers to a connection between scalar and spectral thinking.

1 Ich danke Isabel Mundry, Peter Leu, Jonas Labhart und Christian Strinning für die interessanten, wohlwollenden fachlichen Diskussionen im Entstehungsprozess dieses Aufsatzes sowie der Redaktion der ZGMTH für das intensive Lektorat. Klaus Frieler danke ich für die substanzielle Hilfestellung bei den erforderlichen Berechnungen. 
,Skalen wie diese selbst, »einerseits Tonbestände, andererseits Komplexe von Tonbeziehungen ${ }^{2}$, auf die in Komposition und musikalischer Analyse häufig Bezug genommen wird. Doch eine umfassende Systematik zur Ordnung von Skalen ist und bleibt ein musiktheoretisches Desiderat. Der folgende Aufsatz stellt eine von der diatonischen Heptatonik und den Alterationsmöglichkeiten der Harfe ausgehende Systematik von Skalen vor, die als Ordnungssystem von Tonhöhenmaterial weitgehend unabhängig ist von Formen tonalen Zusammenhangs oder der geschichtlichen Entwicklung tonaler Systeme (wie der DurMoll-Tonalität oder dem Modus-System der Vokalpolyphonie).

Von der Pedalharfe wird ausgegangen, weil sie von der diatonischen Heptatonik geprägt ist und ihre Stimmung in Ces-Dur es ermöglicht, mittels Pedalen jede Saite um zwei Halbtöne zu verkürzen bzw. die entsprechenden Töne aufwärts zu alterieren ${ }^{3}$, um so alle zwölf Halbtöne der Oktave zu erreichen. Sich darauf zu beziehen, hat für die Systematisierung von Skalen den Vorteil, dass die Alterationen in eine Richtung gedacht werden können (von `Ces-Dur` aus um zwei übermäßige Primen nach oben) und dadurch zwei Intervallschritte entstehen, die zur Klassifikation genutzt werden können. (Von `C-Dur aus müssten zwei alterierende Verfahren in verschiedene Richtungen gedacht werden.) Das heißt, es wird aus systematischen Gründen ein instrumentenbautechnischer Umstand als Referenz für ein musiktheoretisches Konzept gewählt ${ }^{4}$, die zu entwickelnde Systematik aber von der etablierten Notation und den entsprechenden Alterationsoperationen abgeleitet.

Nach einleitenden Begriffsklärungen und der Vorstellung bisheriger Ansätze zur Ordnung von Skalen wird in vier Schritten eine Systematik diatonischer Skalen entwickelt, dann werden die Grenzen dieser Systematik aufgezeigt und abschließend ein Ausblick gegeben.

\section{BEGRIFFSBESTIMMUNGEN}

\section{Skala, Tonleiter, Modus}

Die Begriffe `Skalas, sTonleiter und `Modus` werden in der Literatur verschieden gebraucht, zum Teil synonym, zum Teil zur Bezeichnung unterschiedlicher Sachverhalte. Der folgende Text verwendet den Begriff sSkalas, der daher in Abgrenzung zu den beiden verwandten Begriffen näher bestimmt werden soll.

2 Dahlhaus 1998, 624. „Um die beiden Momente, das Material und die Struktur, voneinander zu trennen, bezeichnen manche Autoren den Tonbestand als Tonsystem und das sprincipe régulateur des rapportsı (Fétis) als Tonalität.« (ebd.; Hervorhebungen original) Vgl. auch Hyer 2001a, 509: »key as a musical container«.

3 Bei der Harfe wird die Grundposition Ces-Dur mit b-Vorzeichen gekennzeichnet (z.B. Cb), die erste Erhöhung einer Saite um eine übermäßige Prime (klanglich entsprechend einem Halbton) mit Auflösungszeichen (z.B. C ), die zweite Erhöhung, also insgesamt eine Erhöhung um eine doppelt übermäßige Prime (klanglich entsprechend einem Ganzton), mit \#-Vorzeichen (z.B. C\#).

4 Dieser Vorgang und Analogieschluss ist insofern und insbesondere bei der Harfe gerechtfertigt, als sie bereits seit der Antike als eines der relevanten skalierten Instrumente gelten kann, durch die das mitteleuropäische musiktheoretische Denken geprägt ist. 
»Die Tonleiter, insbesondere die Durskala ${ }^{5}$ gilt [bzw. galt] als paradigmatisches Modell für die Darstellung der Tonart und deren tonale Bezüge [...]. [...] in der Theorie des 18. Jahrhunderts wurde Tonart mit den Verhältnissen in der Tonleiter erklärt und definiert ${ }^{6}$. Weil es in der vorzustellenden Systematik aber nicht um ein tonartbestimmendes Moment, sondern um die abstrakte Klassifizierung von Tonmaterial gehen soll, wird die aufsteigende >Leiter zwar als etablierte und anschauliche Notationsform aufgegriffen, der Begriff `Tonleiter jedoch gemieden.

Der Begriff >Modus` wird aktuell als komplexe und umstrittene Kategorie wahrgenommen; über seine historische und systematische Bedeutung wird intensiv diskutiert. ${ }^{7}$ Markus Jans schreibt dazu: "Was Modus ist, kann weder für die Ein- noch für die Mehrstimmigkeit generell bestimmt werden ${ }^{8}$, "sondern nur nach Zeit und Stil, Gattung und Form «" ${ }^{9}$. Der umfassende Artikel »Mode« in der zweiten Auflage des New Grove Dictionary gibt zum historischen Bedeutungswandel des Begriffs den besten Überblick. ${ }^{10}$ Verhandelt wird u.a., inwiefern und in welchen Zusammenhängen Modi bzw. ModusSysteme als »theoretische[.], präskriptive[.] « oder als "pragmatische[.], deskriptive[.] «"11 Konstrukte verwendet wurden und werden. Interessanterweise lassen sich sowohl der präskriptive als auch der deskriptive Charakter von Modi in Olivier Messiaens Modusbegriff finden: Messiaens bekannte Systematik der »Modi mit begrenzter Transponierbarkeit « ${ }^{12}$ hat einerseits normativen Charakter, insofern es sich um dem Kompositionsprozess vorausgehende abstrakte Setzungen des zu verwendenden Tonmaterials handelt; andererseits stellt Messiaen in seinem Traité de rythme, de couleur et d'ornithologie diese Modi als musiktheoretisches Konzept vor, beschreibt ihre (farblichen) Eigenschaften und verwendet sie deskriptiv, indem er sie analytisch auf die Musik anderer Komponisten anwendet. ${ }^{13}$ In einer auf Messiaen zurückgehenden terminologischen Tradition ließe sich der Begriff >Modus` nun für das hier verfolgte Projekt einer systematischen, dezidiert

5 Vgl. Heygster/Grunenberg 1998, 10: „Die Tonleiter ist eine systematische Anordnung der Spannungsverhältnisse. In einer Melodie sind die diatonischen Spannungsverhältnisse nach künstlerischen Grundsätzen zusammengestellt. Eine solmisierte Durtonleiter stellt die Struktur der Spannungsverhältnisse in der Diatonik allgemein dar.«

6 Moßburger 2012, Bd. 2, 1016 (Ergänzung des Verfassers). Vgl. zur Gleichsinnigkeit und Differenz

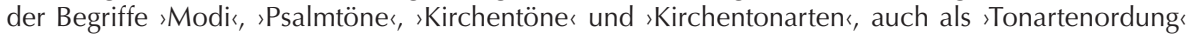
oder `Tonartensystemı, Ziegler 2009 (bezogen auf die Musik im 17. und frühen 18. Jahrhundert auf dem Weg hin zur Dur-Moll-Tonalität).

7 Vgl. Calella 2005, 87; Wiering 2001; Brieger 2010a; Brieger 2010b, 18ff. Gissel verwendet für die Übersetzung des Begriffs `modus` das deutsche Wort `Tonart` bezogen auf die Zeit »von Ockeghem bis Palestrina« $(2013,11)$. Vgl. auch Gissels einleitende Ausführungen zum Verständnis der Begriffe smodus` und stonus` in jener Zeit.

8 Jans 2013, 42.

9 Ebd.

10 Powers/Wiering/Porter/Cowdery/Widdess/Davis/Perlman/Jones/Marett 2001. Vgl. außerdem SchmidtBeste 1997.

11 Calella 2005, 87.

12 Messiaen 2012, 458-476; gebräuchlich ist auch der Terminus »Modi mit begrenzter Transpositionsmöglichkeit« (Messiaen 1966, Bd. 1, 56).

13 Vgl. Messiaen 2012, 459-465. Vgl. zu »The Physics of Music and Color« Gunther 2012. 
ahistorischen Ordnung von Tonhöhenmaterial zwar verwenden, dagegen spricht aber eben die historisch vielfältige und dadurch schillernde Ausdifferenzierung des Begriffs. Auch die über einen reinen Skalenbegriff hinausgehenden Aspekte des Modusbegriffs sprechen dagegen, ihn im vorliegenden Zusammenhang aufzugreifen: „What essentially defines the concept of modality is the repetition of patterns. These may be a group of pitches, resonances (the recurrence of a particular frequency) or rhythmic motives that are organised hierarchically. ${ }^{14}$ Die Ausprägung struktureller Muster, beispielsweise durch Gewichtung einzelner Töne (etwa Finalis, Repercussa) oder motivische Wiederholung und Variation steht nach dieser Auffassung im Zentrum des Begriffs `Modus ‘.

Wenn nun heute von einer abstrahierenden Zusammenstellung, Ordnung oder Klassifizierung von Tonmaterial die Rede ist, wird in Anlehnung an die smusical set theorys häufig der mathematisch geprägte Begriff set (`Menge`, `Gruppe`) benutz $\mathrm{t}^{15}$, und es böte sich deshalb an, auch hier den Begriff ıMenge` oder `Tonhöhenmenge Eine Menge ist jedoch eine in sich potenziell unterschiedlich geordnete Anzahl an zusammengefügten Elementen. Für die hier verfolgte Systematisierung wird aber die leiterartige Anordnung von diatonischem Material wesentlich sein, weshalb auf die Verwendung des Begriffs >Menge` verzichtet und stattdessen der Begriff `Skala bevorzugt wird.

Die Schwierigkeit beim Begriff `Skalaı ist, dass für ihn die Bedeutung 'Leiter, Treppe (lat. scala) und nicht >Menge konnotative Tendenz zu Stufendenken, Linearität oder intervallischem Denken besteht - wie im Fall des Begriffs Tonleiter. ${ }^{16}$ Durch die Etymologie des Begriffs wird deutlich, dass 'Skalaı als metaphorisch-begriffliches Modell zu betrachten ist, dem wie anderen Metaphern eine paradoxe Qualität, Zirkularität und Offenheit zu eigen ist. ${ }^{17}$ Wenn das Skalen-Modell auf Musik bezogen wird, dann entsteht als »Moment[.] von Metaphorizität» unweigerlich der kreative »Konflikt», der jeden »Transfer eines Schemas«, jede »Migration von Begriffen $\aleph^{18}$ umgibt. Dem Modell `Skala` haftet von daher eine Unschärfe

14 Richards 2001, [1]. Zudem orientiert sich der allgemeine Sprachgebrauch des Begriffs >Modus` heute weniger an der klassischen Bedeutung »Maß, Maßstab, Art, Weise« (Hüschen 1961, 402; vgl. den

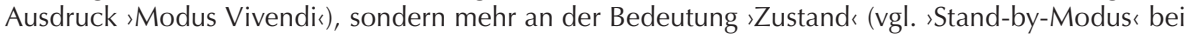
elektrischen Geräten; vgl. »Modes of Vibration and Harmonics«: Gunther 2012, 20), »Permutationsform« (Rambold 2012, 52f.; vgl. auch Kissenbeck 2007, 104) oder »Erscheinungsform[.] " (bei Hüschen 1961, 413, der Josef Rufer zitiert, als Äquivalent zu »Modus« eingeführt; bei Rufer selbst [1966, 78] aber eigentlich direkt bezogen auf »Reihe«). In der neueren Psychotherapietheorie, insbesondere bzgl. der Mentalisierungsbasierten Therapien, sind weitere Moduskonzepte aktuell, die sich auch auf akustische Phänomene anwenden lassen: z.B. Modus als Niveau (vgl. Schrader 2017, 54), Struktur (vgl. Kruse 2017) oder Medium (vgl. Scharff 2010, 14; Schultz-Venrath 2017).

15 Vgl. einführend Scheideler 2005; vertiefend die Darstellung bei Rambold 2012, 20-28. Hingewiesen sei auf das wenig bekannte Book of Modes (1993) des rumänischen Komponisten und Musiktheoretikers Anatol Vieru.

$16 \mathrm{Vgl}$. folgende Bestimmung des Skalenbegriffs: "A sequence of notes in ascending or descending order of pitch. As a musicological concept, a scale is a sequence long enough to define unambiguously a mode, tonality, or some special linear construction« (Drabkin 2001, 366). Vgl. auch Holtmeier 2010a.

17 Vgl. Heinemann 2010, 221.

18 Thorau 2012, 60f., dort im Rahmen einer Darstellung von Nelson Goodmans Symboltheorie. 
an. ${ }^{19}$ Der allgemeine Begriff ,Skalas wird dabei heute verstanden als stufenartig Angeordnetes, als leiterartige Darstellungsform ${ }^{20}$ oder Maß (vgl. sTemperaturskala ). Andere, vor allem musikbezogene Verständnisaspekte bevölkern eine Schnittmenge der beiden Begriffe `Modus` und `Skalas.

Ich verstehe Skalen im Folgenden in pragmatischem Sinn als »Formen elementarer Materialdisposition $\ll^{21}$ und verwende den Begriff unabhängig von der kulturellen Verortung $^{22}$ und den historischen, linearen, harmonischen, räumlichen, farblichen, emotionalen, spektralen oder aufführungspraktischen Implikationen des damit beschriebenen Materials; kurz gesagt: Es geht um diatonische Tonmengen, die in Skalenform dargestellt werden.

\section{Diatonische Skalen}

Das Wort >diatonisch leitet sich aus dem Griechischen ab: diá bedeutet wörtlich »durch $\ll^{23}$, tónos »das, womit man etwas spannt, Seil, Strick, Gurte, [...] Saite« bzw. »Spannung, Nachdruck, Kraft, [...] Hebung der Stimme, Ton, [...] Farbe «24 oder auch »Ganzton « ${ }^{25}$. >Diatonisch bezeichnet vor diesem sprachlichen Hintergrund ${ }^{26}$ in der antiken griechischen Musiktheorie zunächst eines der drei »melodischen Tongeschlechter « ${ }^{27}$ neben schromatisch folgt auf zwei Ganztöne ein Halbton (1-1-1/2). ${ }^{28}$ Zwei disjunkte diatonische Tetrachorde bilden eine Oktave. In einem weiteren Sinne bezeichnet der Begriff >diatonisch dann diejenige Gestalt des über eine Doppeloktav sich erstreckenden tonalen Gesamtsystems, welches ausschließlich aus (disjunkten und konjunkten) diatonischen Tetrachorden zusammengesetzt ist. ${ }^{29}$ Auf diese antike Basis lässt sich ein Großteil der unterschiedlichen Definitionsaspekte von sdiatonisch beziehen, die bis heute den Sprachgebrauch

19 Vgl. Bühler 2014, 7f. und 18-20.

20 Vgl. Schneider 1997, 316, zu »Grundprinzipien jedweder [musikalischen] Skalenbildung«. Vgl. auch Thompson 2013, 127: »The concept of a scale can be defined from physical, mathematical, and psychological perspectives. From a physical perspective, it refers to the set of pitches that can be produced on a musical instrument given a certain tuning system. From a mathematical perspective, one can use a group theoretic description of pitch sets [...]. From a psychological perspective, a scale refers to a mental representation of regularities in pitch that is activated when one listens to music .

21 Eybl 2010, 485.

22 Zum Verständnis von 'Skala` als »Modalleiter« bezogen auf indische rāgas vgl. Michaels 2005, 40; zu arabischen maqamāt vgl. Powers 2006. Vgl. zur »Problematik äquidistanter Stimmungen« Schneider 1997, 332-399 (Zitat: 332).

23 Gemoll 1937, 194.

24 Ebd., 744.

25 Cahn 1995, 1214.

26 Vgl. Rohringer 2010, 82: »>Diatonik» (griech. diátonos: durch Ganztöne gehend)«.

27 Ebd.

28 Vgl. Cahn 1995, 1214 und 1218.

29 Vgl. ebd. 1219. Vgl. auch Vetter 1956, 852-856. 
verschiedener Autoren prägen: ${ }^{30}$ Diatonik im Sinne eines pentatonischen oder heptatonischen »Tonvorrat $[\mathrm{s}]$ « $^{31}$, im Sinne einer Intervallanordung unregelmäßig alternierender Ganzton- und Halbtonschritte ${ }^{32}$, im Sinne eines Systems an Tonbeziehungen ${ }^{33}$, im Sinne von Basistonleiter, Stammtonreihe, Alterationslosigkeit ${ }^{34}$, als »Inbegriff der Modi $\aleph^{35}$. Zur Erklärung der inneren Ordnung diatonischer Zusammenhänge wird in der Regel die Schichtung reiner Quinten herangezogen. ${ }^{36}$

Im Folgenden bezeichne ich mit dem Begriff >diatonisch jene Skalen, die auf der Stammtonreihe ces-des-es-fes-ges-as- $b$ fußen, damit ihrer Herkunft nach minimale interne Quintenbreite besitzen, aus höchstens sieben Tönen bestehen und in der halbtöniggleichstufigen Stimmung transponiert werden können. Ich gehe davon aus, dass die Töne alteriert werden können, ohne dass sie dadurch ihren "Stufenwert ${ }^{37}$ verlieren, dass Töne aber zugleich enharmonisch verwechselbar sind und in der Gleichstufigkeit Intervalle, die enharmonisch verwechselte Töne (z.B. $d$-e; $d$-fes) enthalten, austauschbar sind sowie dass die Oktavidentität gilt. In der Folge verwende ich den Begriff stransheptatonische Skalen` zur Bezeichnung von Skalen, die mehr als sieben Töne aufweisen.

30 Vgl. Cahn 1995; Dahlhaus 1998, 625; Moßburger 2012, Bd. 2, 947-959.

31 Rohringer 2010, 82. Vgl. zur Unterscheidung von »diatonische[r] Pentatonik« und »diatonische[r] Heptatonik« Rambold 2012, 47-58 (Zitate: 47 und 51). Vgl. zu weiterführender Literatur zum Begriff ,Pentatonik« Sprenger 2009. Er unterscheidet »tonikale Pentatonik« (ebd., 293), "subdominantische Pentatonik« (ebd., 294) und »dominantische Pentatonik« (ebd., 295; Hervorhebungen original). »Das Tonmaterial der diatonischen Leiter entspricht der Tonklassenmenge pcs [pitch class set] 7-35" (Rambold 2012, 54; Kursivierung original). Vgl. auch Amon 2005, $298 \mathrm{f}$.

32 Vgl. Rohringer 2010, 82, sowie Petersen 1971, 45; zur Untersuchung intervallischer Strukturen vgl. Rings 2011.

33 Vgl. Heygster/Grunenberg 1998 zu »Tonbeziehungen« und deren »Wirkung« (ebd., 10f.), bzw. deren »diatonischen Affekten« (ebd., 23) als Folge der »diatonischen Spannungsverhältnisse« (ebd., 10). Vgl. auch Lindley/Turner-Smith 1993, 125: »A strictly diatonic scale represents an MT-system with interlocking chains of three major and three minor triads." (Ein >MT-system « wird ebd., 31, als »[a] kind[.] of tempered harmonic system« definiert.)

34 Vgl. Rambold 2012, 58.

35 Dahlhaus 1966, 534.

36 »Als diatonisch gilt ein Tonvorrat, der durch eine lückenlose Reihe von mindestens drei (`Triton`) und höchstens sieben (`Heptaton`) Tönen im Abstand reiner Quinten gebildet wird.« (Rohringer 2010, 82) Dass es sich bei Diatonik »um einen Tonvorrat handelt, dessen Töne sich in eine reine Quintschichtung bringen lassen«, ist "gleichbedeutend mit minimaler interner Quintenbreite" (Kissenbeck 2007, 15; Hervorhebung original). „Die Besonderheit der Pentatonik besteht nun darin, dass sie gerade der größtmögliche Tonvorrat mit minimaler interner Quintenbreite ist, der keine kleinen Sekunden enthält. [...] Die Besonderheit der [heptatonischen] Diatonik besteht [...] darin, dass sie gerade der größtmögliche Tonvorrat mit minimaler interner Quintenbreite ist, der keine benachbarten kleinen Sekunden enthält.« (ebd., 16) Kuhn $(2014,245)$ greift zur Bezeichnung der diatonischen Heptatonik den Begriff `Heptatonia prima sauf.

37 »Alterierungen ändern nicht den Stufenwert« (Schönberg 1957, 40). Vgl. Holtmeier 2010b, 89f. Béla Bartók (1976, 367) schreibt: »In our polymodal chromaticism, however, the flat and sharp tones are not altered degrees at all; they are diatonic ingredients of a diatonic modal scale." Kuhn (2014, 256) äußert bezogen auf Bartóks Musik: »Es gibt keine Unterscheidung zwischen diatonischen und alterierten Stufen.« Krüger $(2011,87)$ versteht unter »Stufendeckung» den »Anteil der verwendeten Stufen in Bezug auf die Gesamtzahl der Stufen einer Skala». 


\section{Chromatische Skala}

Der Begriff >diatonisch`muss, um selbst bestimmt zu sein, nun zu komplementären Begriffen wie schromatisch` ins Verhältnis gesetzt werden. ${ }^{38}$ Die Basis für diese Unternehmung ist jedoch insofern problematisch, als die Begriffe bei verschiedener Bedeutung in verschiedenen Kontexten unterschiedlich ins Verhältnis gesetzt wurden und zudem auf Unterschiedliches bezogen wurden und werden. So wird der griechische Begriff chroma (Farbe) in der Musik heute bezogen auf Töne (chromatische Versetzungen eines diatonischen Tones), Intervalle (diatonische und chromatische Intervalle), Akkorde (chromatische Akkorde) oder Skalen (chromatische Skala). Ich begnüge mich hier mit der Diskussion einzelner Aspekte der chromatischen Skala. Für nähere Informationen zum Thema der Chromatik verweise ich auf einschlägige Fachpublikationen. ${ }^{39}$

Die entscheidende Frage für unseren Zusammenhang ist nun: Gibt es nur eine chromatische Skala oder mehrere $?^{40}$ Zumeist wird von $>$ der chromatischen Skala als einer einzigen Skala mit zwölf gleichstufigen Halbtonschritten gesprochen, die keinen Grundton besitzt und damit als Materialskala angesehen werden kann, aus der Reihen oder andere Gebrauchsskalen gebildet werden können. ${ }^{41}$ Es wäre nun aber auch möglich, bestimmte, von dieser chromatischen Skala abgeleitete Skalenausschnitte als chromatische Skalen zu bezeichnen. `Diatonisch und `chromatisch` wären dann qualifizierende Begriffe, die eine bestimmte Quantität wie die Heptatonik näher beschreiben würden. Anatol Vieru bestimmt die Qualitäten einer Skala mithilfe eines Maßes der >diatonischen und chromatischen Kapazität (»diaton[ic] and [...] chromatic capacity « ${ }^{42}$ ). Er nennt das

38 Rohringer $(2010,82)$ definiert ıDiatonikı dem Verständnis »heutiger Theorie« gemäß als Tonvorrat, zu dem `Chromatikı und `Enharmonikı relationale Begrifflichkeiten darstellen: Chromatik und Enharmonik versteht er als eine Erweiterung der Diatonik durch »zunehmende[.] Quintenbreite«, erst durch weitere fünf, dann weitere zwölf Töne im Quintabstand. Kuhn verwendet in Bezug auf die Musik Béla Bartóks die Negation ınicht-diatonischı (vgl. Kuhn 2014, 248). Bartók selbst (1976, 363) nennt die Skalen $g$-a-b-c-des-es-f / g-as-b-ces-des-es / g-a-b-cis-d-e-f. Kuhn $(2014,248)$ erklärt solche Skalen bei Bartók »als Mischung diatonischer und nichtdiatonischer Skalensegmente» (vgl. auch Antokoletz 1984, 205). „As the result of superposing a Lydian and Phrygian pentachord with a common fundamental tone, we get a diatonic pentachord filled out with all the possible flat and sharp degrees.« (Bartók 1976, 367) Laut Kuhn (mit Verweis auf László Somfai) komme Bartók so zu einer »Heptatonia secunda« (Kuhn 2014, 249; der zitierte Begriff nach Lajos Bárdos). Vgl. auch Petersen 1971, 45.

39 Vgl. Cahn 1995 und Rohringer 2010. Zu »Affekt und Chromatik« bzw. zu »chromatischen Effekten« in der Musica ficta vgl. Menke 2015, 308-313 (Zitate: 308 und 310).

40 Vgl. den Plural von Chromatik bzw. verschiedene Chromatiken wie die `Rückungschromatikı bei Schütz, die ıvagierende Chromatikı bei Wagner oder die `Farbchromatikı im Impressionismus. Bartók unterscheidet »three chromatic forms « (Gilles 1982, 121): »bi-modality or polymodality«, »modal chromaticism« und »melodic new chromaticism« (Bartók 1976, 376). Ergänzend dazu Ricarda Rätz $(2009$, 101) mit Bezug auf »Josef Matthias Hauers System der Tropen«: »Tropen sind Zwölftonskalen, die aus zwei sich im Tonvorrat zu 12 verschiedenen Tönen ergänzenden Hexachorden bestehen."

41 Voraussetzung dieser Bestimmung ist, dass das Verhältnis von Stimmungssystem zu Tonstufen im Sinne der Gleichstufigkeit geklärt ist. Vgl. das im folgenden Abschnitt genannte Vorgehen von Schneider 1966.

42 Vieru 1993, Bd. 1, 96 (Ergänzung des Verfassers). 
$\mathrm{Maß}$ »DIACRO $«^{43}$ und entwickelt es als Bruch CRO/DIA: im Zähler: `Chromatizität` (Anzahl an nicht unterbrochenen Sekundreihen), im Nenner: ‘Diatonizität (Anzahl an nicht unterbrochenen Quintenreihen). Vieru geht dabei von der Mengenlehre aus, in ähnlicher Weise wie die US-amerikanische ımusical set theory`, und bezieht sie auf Ton- und Intervallmengen.

Ich will hier am Sprachgebrauch einer einzigen chromatischen Skala festhalten, die Möglichkeiten der qualifizierenden Begrifflichkeiten im Gedächtnis behalten und eine Klassifikation entwickeln, die ihren Ausgangspunkt nicht bei Tonstufen oder Intervallen, sondern bei den Akzidenzien nimmt - so wie diese in der skalaren Anordnung der Harfe erscheinen und in unserer Notation funktionieren. Damit ist deutlich, dass die unten generierten Tonvorräte ihrer Herkunft nach diatonisch sind und in Entsprechung zu dieser Perspektive allgemein als >diatonische Skalen` benannt werden können.

\section{BISHERIGE ANSÄTZE ZUR SYSTEMATISIERUNG VON SKALEN}

Musiklexika wie The New Grove Dictionary, Die Musik in Geschichte und Gegenwart oder das Lexikon der Systematischen Musikwissenschaft lassen eine Systematisierung von Skalen weitgehend vermissen. Zu finden sind vorwiegend Beschreibungen von Begriffen, die das musikalische Verständnis von Skalen mitbedingen. ${ }^{44}$ Ansätze zu einer geordneten Darstellung von Skalen haben den Charakter von Entwürfen, Gruppierungen oder Sammlungen.

Carl Dahlhaus schlüsselt in der ersten Auflage der Musik in Geschichte und Gegenwart verschiedene Verständnisweisen des Begriffs `Tonsystem` als Tonvorrat, Stimmung, Schema von Tonbeziehungen oder Melodiestrukturen auf, weist auf Verwirrungen und Unzulänglichkeiten der Terminologie hin und erläutert den Begriff `Tonsystem` im Hinblick auf europäische Musik. ${ }^{45}$ In einer außereuropäische Tonsysteme darstellenden Tafel zeigt Marius Schneider im Rahmen desselben Artikels listenartig angeordnete Skalen und Melodiebeispiele unter Verwendung der zwölftönigen chromatischen Skala. ${ }^{46}$ Diese geordnete Liste von möglichst vorzeichenlos geschriebenen Skalen, ausgehend von den Stammtönen, macht anschaulich, dass durch Auswahl von Tönen aus der gleichstufigen Chromatik verschiedenste Skalen gebildet werden können. Diese Systematisierungsmöglichkeit, ihre Vorteile und Schwierigkeiten werden von Schneider aber nicht weiter ausgeführt.

Auch im Jazz, in dem sehr differenziert mit Skalen gearbeitet wird, gibt es Bemühungen, Skalen geordnet darzustellen. Andreas Kissenbeck z.B. zeigt im ersten Band

43 Ebd.

44 Vgl. z.B. New Grove Dictionary (2. Auflage): »Key (i)« (Hyer 2001a), »Mode« (Powers/Wiering/ Porter/Cowdery/Widdess/Davis/Perlman/Jones/Marett 2001), „Scale« (Drabkin 2001), »Tonality» (Hyer 2001b); MGG (1. Auflage): »Ton« (Reinecke/Winckel 1966), »Tonalität« (Dahlhaus/Reinecke/ Schneider 1966), »Tonsysteme« (Dahlhaus 1966 und Schneider 1966); MGG (2. Auflage): »Ton« (Rettelbach 1998), »Tonalität» (Dahlhaus 1998), »Modus III« (Schmidt-Beste 1997).

45 Vgl. Dahlhaus 1966.

46 Vgl. Schneider 1966, Ausfaltblatt mit fünf Seiten zwischen den Spalten 544 und 545. 
seiner Jazztheorie, wie Skalen in Bezug zu Akkorden bestimmt und verwendet werden können. ${ }^{47}$ Er geht dabei auf die in der Jazz-Improvisation gängigen Skalen ein, darunter »Bebop-Skalen « ${ }^{48}$ und `Blues-Tonleitern`. Als »Modi« von »Harmonisch-Moll (HM)«, »Melodisch-Moll (MM) « und »Harmonisch-Dur (HD) « bezeichnet Kissenbeck die auf den verschiedenen Skalentönen beginnenden Rotationen dieser Skalen, z.B. HM ${ }^{1}$ bis $\mathrm{HM}^{7}{ }^{49}$ Anschließend stellt er symmetrische Skalen wie die »Messiaen-Skalen «50 dar, um abschließend auf »Exotische Skalen $\aleph^{51}$ einzugehen. Er betont: »ede Tonleiter besitzt eine klangliche Charakteristik. Sie wird wesentlich von ihrer Intervallstruktur (u.a. Tritonusgehalt) sowie auch von ihrer internen Quintenbreite [...] bestimmt. «52 Und weiter: »Die Zuordnung einer Skala zu einem Akkord klärt die klangliche Bedeutung aller zwölf Töne in Bezug auf die jeweilige harmonische Situation « ${ }^{53}$. Kissenbeck gibt damit für die praktische Jazz-Improvisation eine sinnvolle Anleitung, doch unternimmt er, abgesehen von einer Gruppenbildung, keine systematisierende Klassifizierung von Skalen.

Eric Krüger analysiert und beschreibt ähnliche Skalengruppen wie Kissenbeck, aber nun nicht als Anleitung zur Jazz-Improvisation, sondern mit systematisierenden Ansätzen insbesondere hinsichtlich der »Anzahl der Stufen«, der »Intervallverhältnisse« und der »Lage der unterschiedlichen Stufenintervalle $\ll^{54}$. Jeder Analyse stellt er ein »Skalendiagramm [.] zur Visualisierung “ ${ }^{55}$ der jeweiligen Skalen-Architektur zur Seite. Diese Darstellung macht den Hauptteil des für die hier behandelte Thematik informativen Buchs aus. Ein klanglicher Vergleich der Skalen ergänzt die Beschreibung, aber eine ausgearbeitete Systematisierung fehlt auch hier.

Stefan Rohringer zeigt im Artikel »Diatonik/Chromatik/Enharmonik» im Lexikon der Systematischen Musikwissenschaft den Entwurf einer umfassenden Systematisierungsmöglichkeit von Skalen: Eine »einfach-tritonische Heptatonik « ${ }^{56} F-c-g-d^{1}-a^{1}-e^{2}-h^{2}$ bildet zwischen unterstem und oberstem Ton einen Tritonus. »Dieses einfach-tritonische Hep-

47 Vgl. Kissenbeck 2007. Ähnlich Haunschild (1997, 70): »[Die] Akkord-Skalen-Theorie [...] besagt, daß zwischen Akkorden und den dazu passenden Skalen im Prinzip nicht unterschieden werden muß, da sie ja beide aus denselben Tönen bestehen. Akkorde und Skalen werden also gleichberechtigt behandelt. Akkorde werden als vertikale Klanggebilde (meist in Terzschichtung) angesehen, die sich in Form von Skalen in horizontaler Anordnung (im Sekundabstand) darstellen lassen«.

48 »Die Grundidee der Bebop-Skalen besteht [...] darin, die[.] Struktureigenschaft des Abwechselns zwischen Akkord- und Durchgangstönen auf die gesamte Tonleiter auszuweiten." (Kissenbeck 2007, 94)

49 Siehe unten, Abschnitt `Harmonisch-Molk (HM), 'Melodisch-Molk (MM), 'Harmonisch-Dur (HD). Vgl. Kissenbeck 2007, 104-107.

50 Ebd., 111-114.

51 Ebd., $116 f$.

52 Ebd., 101. Vgl. auch Haunschild 1997, 18ff. Nach Kissenbeck (2007, 14) "wird das Klangbild von Tongruppen maßgeblich dadurch mitbestimmt, wie eng die beteiligten Töne im Quintenzirkel beieinanderliegen. Man spricht in diesem Zusammenhang von der sinternen Quintenbreite، (Maßeinheit: $\mathrm{QB}) . «$ Als Beispiele nennt Kissenbeck (ebd., 15) »g-c-f (2 QB) und gis-c-e (8 QB) «.

53 Ebd., 9.

54 Krüger 2011, 15.

55 Ebd., 17.

56 Rohringer 2010, 83. 
taton wird infolge der symmetrischen Umlagerung des mittleren Tons durch zwei verminderte Quinten zum doppelt-tritonischen (e-b, fis-c) [Fis-c-g- $\left.d^{1}-a^{1}-e^{2}-b^{2}\right]$ bzw. dreifachtritonischen (es-a, f-h, g-cis) [ $F$-cis-g- $\left.d^{1}-a^{1}-e s^{2}-h^{2}\right]$ Heptaton. Bestimmt man den mittleren Ton des Heptatons zum Anfangston einer Grundskala«, können verschiedene Skalen gebildet werden, darunter die »Kirchentonleitern « und die »melodische Molltonleiter ${ }^{57}$. »Als Grenzfälle der Diatonik können Tonvorräte gelten, in denen einzelne Quinten des Heptatons in asymmetrischer Verteilung vermindert sind. ${ }^{58}$ Diese Bestimmungen können einerseits aufgegriffen werden, weil sie einen Anhaltspunkt dafür bieten, dass durch Veränderung des Tritonusgehalts bzw. durch Alterationen Skalen klassifiziert werden können; auch dass es "Grenzfälle der Diatonik« gibt, ist ein unbedingt bedenkenswerter Hinweis; andererseits ist das beschriebene Vorgehen nicht bis zu einer systematischen Klassifikation ausgeführt.

Als Spezialfall sei hier noch der kompositorische Versuch von Urmas Sisask genannt, den klanglichen Charakter einer einzigen Skala in vielfältigen Konkretisierungen vorzuführen. Seine 24 Hymns für Chor basieren ausschließlich auf der >Planeten-Skalaı cis- $d$ fis-gis-a. ${ }^{59}$ Auf deren Grundlage und durch die differenzierte Verwendung von Rhythmus und Dynamik erschafft er eine Klangwelt, die an eine einzige Farbe oder einen einfarbigen Raum erinnert. Eine Klassifizierung von Skalen wurde von Sisask meines Wissens nicht ausgearbeitet.

\section{ENTWICKLUNG EINER SYSTEMATIK DIATONISCHER SKALEN}

\section{Subtraktive Skalen}

Aus der diatonischen Stimmung der Pedal-Harfe in Ces-Dur (sieben Saiten pro Oktave) und ihrer Möglichkeit, den Klang jeder Saite mittels je eines der sieben Pedale um eine oder zwei übermäßige Primen (im klanglichen Ergebnis: Halbtonabstände) zu erhöhen, entlehne ich, wie bereits erwähnt, die Idee, diatonische Skalen durch ein zunächst subtraktives und dann additives Verfahren zu systematisieren. Die dabei zum Einsatz gelangenden Vorzeichen werden gemäß ihrer Positionierung im Tonraum bzw. ihrem Auftreten in der herkömmlichen Generalvorzeichnung nummeriert ( $b$ und fis als 1., es und cis als 2. b- bzw. \#-Vorzeichen usw.; Bsp. 1).

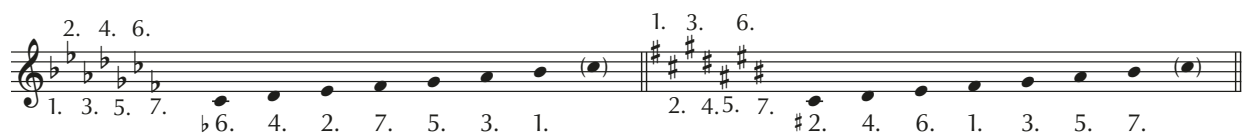

Beispiel 1: Nummerierung der Vorzeichen

57 Ebd. (Ergänzungen des Verfassers).

58 Ebd. Hier nennt Rohringer z.B. die Skala >Mi-Sheberach`d-e-f-gis-a-h-c-d.

59 Vgl. Sisask 2005. Vgl. auch die modalen Sätze, die Rambold (2012, 325-362) zu verschiedenen Grundtonmengen abdruckt. 
Zunächst werden nun die sieben b-Vorzeichen der Ces-Dur-Skala sukzessive ıweggestrichen (dieses Verfahren nenne ich ssubtraktiv`), was klanglich gleichbedeutend ist mit der sukzessiven Erhöhung der Skalentöne um einen Halbton, sodass am Ende dieses Verfahrens die C-Dur-Skala steht. Insgesamt entstehen dadurch 128 subtraktive Skalen (Bsp. 2 und Anhang/Bsp. 12). ${ }^{60}$ Diese 128 Skalen umfassen alle «Kirchentonarten andere im Jazz etablierte Skalen wie die salterierte Skalas $\left(=M^{7}\right)^{61}$ oder die `Ganztonskalas, aber interessanterweise auch viele Skalen, die keinen Namen tragen. Man kann diese Skalen unterteilen in kontinuierlich alterierte Skalen (ein Vorzeichen nach dem anderen wird weggestrichen) und diskontinuierlich alterierte Skalen (z.B. erstes und fünftes Akzidens werden weggestrichen). In der europäischen Tradition wurden hauptsächlich die auf kontinuierlicher Alterierung basierenden Skalen verwendet: So entstehen die `Kirchentonarten<, wenn die b-Vorzeichen nacheinander subtraktiv aus der Ces-Dur-Skala weggestrichen werden. ${ }^{62}$ Die Skalen mit diskontinuierlicher Alterierung können aufgrund der entstehenden übermäßigen Sekunden bzw. kleinen Terzen - unter Vernachlässigung von Stimmungsdifferenzen - mit arabischen bzw. asiatischen Skalen assoziiert werden; sie sind gemäß der obigen Definition aber ebenfalls diatonische Skalen. Beachtenswert ist, dass durch das subtraktive Verfahren neben der bekannten anhemitonischen Pentatonik auch hemitonische pentatonische Skalen entstehen (z.B. durch Wegstreichen des ersten, zweiten, vierten und fünften b-Vorzeichens; b1.2.4.5.: ces-d-[e]-fes-g-as-[h]). Als `Grundgestalt` der diatonischen Skala kann `lonisch`bzw. die Dur-Skala gelten, weil ihre Intervallverhältnisse mit denjenigen der Stammtonreihe identisch sind.

Skalen, die (ausgehend von der Ces-Dur-Skala) nur b-Vorzeichen und --Zeichen enthalten, sind, wie aus dem Vorangehenden folgt, subtraktive Skalen; sie können auch Skalen ersten Grades oder Skalen der ersten `Halbtonerhöhung` genannt werden.

60 Diese Anzahl kann mithilfe des Pascal'schen Dreiecks errechnet werden, wenn in der siebten horizontalen Zeile des Dreiecks (oberste Zeile $=0$. Zeile) die Zahlen addiert werden $(1+7+21+35+35+21+7+1=128)$. Die Summen im Pascal'schen Dreieck entsprechen den Quadratzahlen, hier $2^{7}=128$.

61 Melodisch-Moll vom 7. Skalenton aus (Rotation der Töne).

62 Wegstreichen der b-Vorzeichen unter numerischer Angabe von deren jeweiliger Position (zur Erklärung der Nomenklatur siehe unten, Abschnitt Nomenklatur 1: Aspekte des subtraktiv-additiven Alterationsverfahrens): Ionisch b0.; Lydisch b7.; Lokrisch b6.7.; Phrygisch b5.6.7.; Äolisch b4.5.6.7.; Dorisch b3.4.5.6.7.; Mixolydisch b2.3.4.5.6.7.; Ionisch b1.2.3.4.5.6.7. Das Ergebnis sind Skalen, die auf den folgenden Stufen Auflösungszeichen tragen: Ionisch: ६0; Lydisch: ६4; Lokrisch: Ł14; Phry-

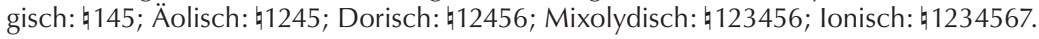




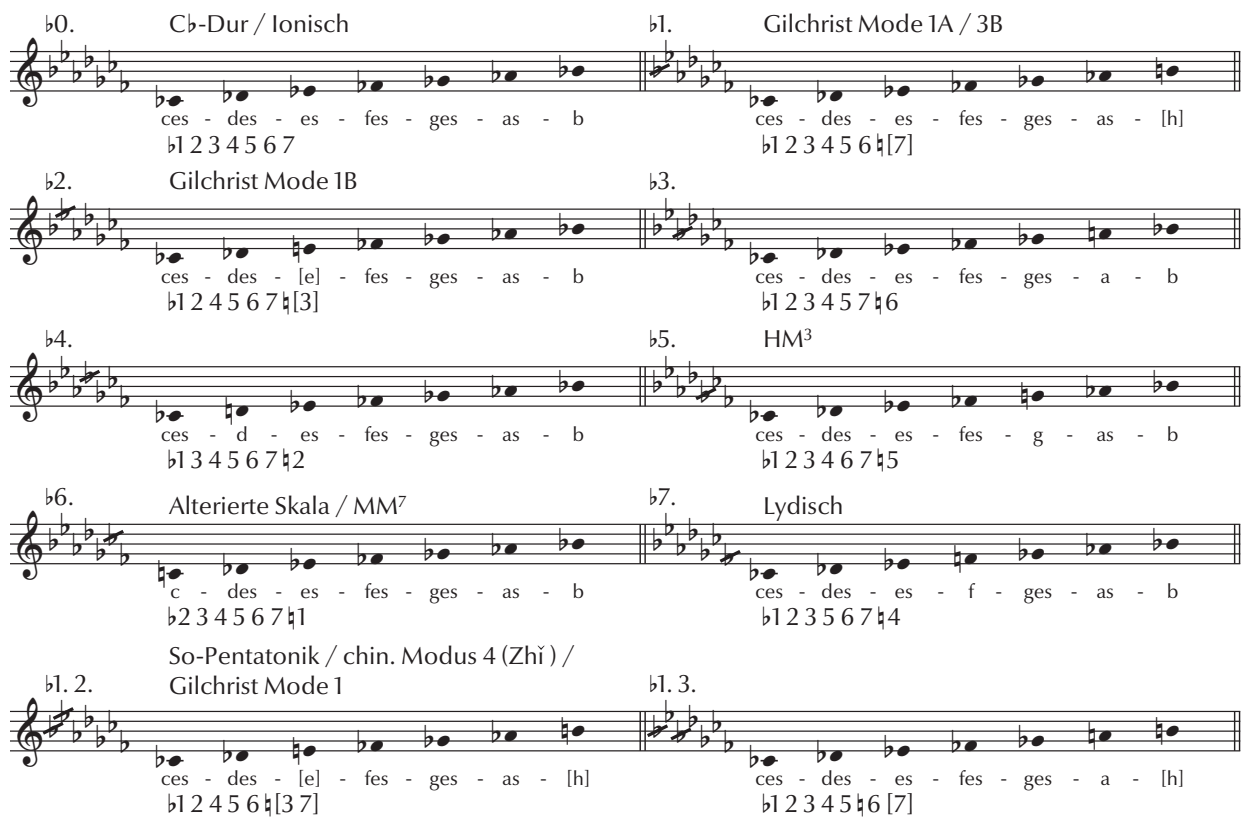

Beispiel 2: Subtraktive Skalen 1-10 (vollständige Auflistung aller 128 Skalen im Anhang/Beispiel 12) ${ }^{63}$

\section{Additive Skalen}

Nun gibt es aber weitere diatonische Skalen wie das sogenannte `Zigeuner-Molk (c-d-esfis-g-as- $h$ ), das iZigeuner-Dur (c-des-e-f-g-as- $h$ ), die senigmatische Skala ${ }^{64}$ (c-des-e-fisgis-ais-h) oder der Klezmer-Musik zuzuordnende Skalen wie `Mi-Sheberach (c- $d$-es-fis$g-a-b)^{65}$, die sich nicht allein auf subtraktivem Weg bilden lassen, sondern eines zweiten

63 Zur Bedeutung der Ziffernreihen unter den Systemen siehe unten, Abschnitt Nomenklatur 1.

64 Der Begriff sscala enigmaticas tritt an prominenter Stelle zum ersten Mal im Untertitel zu Giuseppe Verdis Ave Maria. Scala enigmatica, armonizzata a quattro voci miste, sole (Quattro pezzi sacri Nr. 1; Erstpublikation 1898) auf (vgl. Schweikert 2001, 505).

65 Vgl. Bauer 1999, 45. Vgl. auch Bauer 2002, Kap. 2 »Klezmology. Typische Tonskalen«, sowie Rohringer 2010, 83. Schleifer $(2001,56)$ schreibt: "Despite various endeavours to describe some of the shteygers, a comprehensive theory of the Ashkenazi synagogue modes is still lacking." Er beschreibt neben anderen Skalen auch "the mi shebberakh (or av harahamim) shteyger, which is based on the so-called Ukrainian-Dorian scale, that is, the Dorian scale with a raised 4th degree« (ebd., 57; Hervorhebungen original). Idelsohn $(1992,25)$ betont: »As a result of the quarter tones there are a great variety of scales in existence «, und fährt fort: »[...] in the Orient the Jews sing in those scales, using the quarter-tone steps of their neighbors, while [...] the Jews of the Occident employ the same scales with steps of the semi-tone system « (ebd., 26; Hervorhebungen original). Später beschreibt Idelsohn die Bildung der Skalen aus Tetrachorden und ihre Beziehung zu den ımodes`; 'Mi-Sheberach « wird dabei nicht erwähnt, besitzt aber Ähnlichkeit zur zweiten der folgenden Skalen: "Synagogue song 
Schritts, der Addition, bedürfen. Addition bezeichnet hier das Hinzufügen von \#-Vorzeichen nach vorangegangener Subtraktion von b-Vorzeichen. Das additive Verfahren entspricht also bei gleichstufiger Stimmung einer , Ganztontransposition` ausgehend von der Ces-Dur-Skala (siehe Bsp. 3 und unten den Abschnitt Nomenklatur 1). So entsteht zum Beispiel: ıZigeuner-Molk (c- $d$-es-fis-g-as- $h$ ) durch den Alterationsprozess b1.4.5.6.7. \#1. Die Skalenstufen zeigen als Ergebnis folgende Alterationen: $1 \natural, 2 \hbar, 3 b, 4 \sharp, 5 \hbar, 6 b, 7 \hbar$; das heißt: Die entstehenden Skalen müssten ssubtraktiv-additive Skalen senannt werden, sollen aber, der Einfachheit halber, sadditive Skalen`, sSkalen zweiten Grades` oder 'Skalen der zweiten Halbtonerhöhung، heißen (Bsp. 3). Jede der subtraktiven Skalen kann also theoretisch mit den sieben \#-Vorzeichen additiv bearbeitet werden, wodurch eine schier unüberblickbare Anzahl an möglichen Skalen entsteht (insgesamt 128x128 = 16.384). Manche dieser Skalen sind in Bezug auf die Intervallverhältnisse mit einer subtraktiven Skala identisch (z.B. Ganztonskala als subtraktive Skala: ces-des-es-f-g-a-[h] bzw. c-d-e[fes]-ges-as-b; als additive Skala: $c$-d-e-fis-gis-ais-[his]), andere sind neu (Bsp. 3).

b1. 4.5.6.7. \#1. 'Zigeuner-Molk

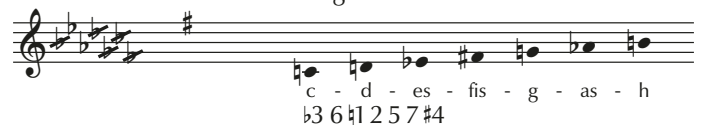

b3. 4. 5. 6. 7. $\sharp 1$.

b3 6 的 $257 \# 4$

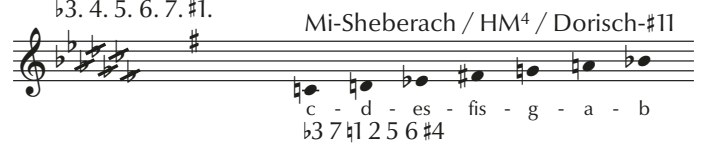

b1. 2. 3. 5. 6. 7. \#1.3.5. Enigmatische Skala

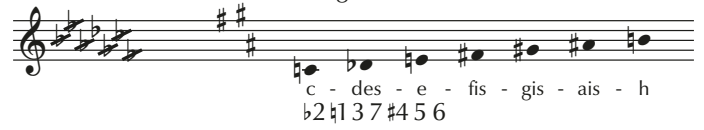

b2. 3. 4. 5.6.7. $\sharp 1 . \quad M^{4} /$ Mixolydisch- $\sharp 11$

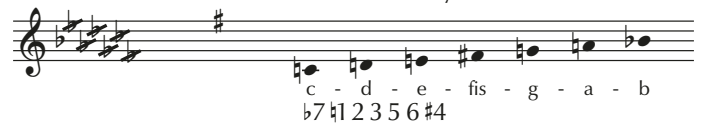

b1. 3. 4. 5.6.7. $\sharp 1 . \quad \mathrm{HD}^{4} /$ Lydisch-Moll

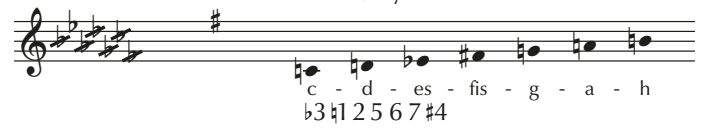

Beispiel 3: Additive Skalen (Auswahl)

is based upon three principal scales. First, the tetrachord e-f-g-a, conjunctively repeated: e-f-g-a$b^{b}$-c-d becomes a heptachord, while disjunctively: e-f-g-a+b-c-d-e constitutes an octachord. Out of this scale the Ashkenazim developed the scale of the Adonoy-Moloch mode, by adding two tones below the tonic $(d-c)$ and by lowering e in the octave $\left(e^{b}\right)$, thus creating the following succession of notes: $c-d-e-f-g-a-b^{b}-c-d-e^{b}$. The second scale consists of the minor tetrachord $d-e-f-g$, which, if conjunctively repeated: $d-e-f-g-a-b^{b}-c$, produces a heptachord, and by its disjunctive repetition: $d$-e-f$g+a-b-c-d$, forms an octachord. The third scale: $d-e^{b}-f^{\sharp}-g+a-b^{b}-c-d$, the basis for the Ahavoh-Rabboh mode, is formulated of two disjunctive tetrachords." (ebd., 478; Hervorhebungen original) 
Aufgrund der beschriebenen Tatsache, dass identische Skalen (wie im Fall der Ganztonskala) auf unterschiedlichen Wegen gebildet werden können, wird es wichtig, Regeln für die Darstellung, Benennung und Kategorisierung einer Skala aufzustellen: Wenn zwei unterschiedliche Darstellungen einer Skala (quasi Transpositionen) möglich sind, soll die jeweils seinfachere`, durch weniger Operationen von der Grundskala Ces-Dur ausgehend erreichbare, zur Benennung gelten. Es stellt sich die Frage, wie viele tatsächlich verschiedene Skalen vor dem Hintergrund dieser systematischen Einschränkung mithilfe der beiden Alterationsarten überhaupt gebildet werden können. Eine mathematisch exakte Berechnung ergibt, dass nach Ausschluss von `Duplikaten` insgesamt 977 Skalen verbleiben, bei denen jeder Stammton entweder mit b-, $\mid$ - oder \#-Vorzeichen versehen ist. 359 dieser Skalen sind heptatonisch; durch alterierte Stammtöne, die bei enharmonischer Verwechslung mit unmittelbar benachbarten Skalentönen zusammenfallen, entstehen außerdem 415 hexatonische, 178 pentatonische und 25 tetratonische Skalen. ${ }^{66}$

\section{Nomenklatur 1: Aspekte des subtraktiven bzw. additiven Alterationsverfahrens}

Einer der wichtigsten Aspekte des dargelegten subtraktiven bzw. additiven Verfahrens ist, dass auf der Ebene der Vorzeichen und nicht auf der Ebene der Stufen operiert wird. Es wurde ein Prozedere gewählt, das nacheinander zwei ^Halbtontranspositionen` nach oben zulässt und nicht eines, das von drei verschiedenen Zuständen einer `Stufe`, die mit denen der Nachbarstufen überlappen, ausgeht. Die Konsequenz ist, dass nicht allein die konkrete Skala als Ergebnis eines Alterationsprozesses von Stufen, sondern auch der Prozess der Alterierung selbst benannt werden muss.

Eine erste Nomenklatur bezieht sich daher auf den Prozess der Alterierung. Er kann dargestellt werden, indem zunächst ein $b$ geschrieben und danach die Nummern der $b$-Vorzeichen, separiert durch Punkte, notiert werden. Die Nummerierung der Vorzeichen orientiert sich dabei an der Reihenfolge ihres Auftretens in der Generalvorzeichnung (Bsp. 1). So entsteht z.B. Phrygisch c-des-es-f-g-as-b durch: b5.6.7. (das fünfte, sechste und siebte $b$ wurden weggestrichen, d.h. durch ein $h$-Zeichen ersetzt). Bei additiven Skalen sind \#-Vorzeichen zu notieren, wo vorher bereits ein $b$ weggestrichen worden war. Zur Bezeichnung des additiven Prozesses werden die \#-Vorzeichen entsprechend angefügt, z.B.: b7. \#1. (das siebteb wurde zunächst weggestrichen [aus dem Ton fes resultierte ein $f$ ] und auf der gleichen Stufe dann das erste \# zugefügt, sodass sich ein fis ergibt); die Skala, die entsteht, ist: ces-des-es-[fis]-ges-as-b. Eine Schwierigkeit dieser Nomenklatur besteht in der unterschiedlichen Zuordnung von Ziffern zu den einzelnen Skalenstufen

66 Geht man zur Berechnung (mithilfe von pitch classes und Kombinationen von Intervallzahlen) davon aus, dass jede Stufe in drei `Alterationszuständen $(b, \natural, \sharp)$ auftreten kann, entstehen $3^{7}=2187$ Skalen. Darunter sind dann doppelt und mehrfach auftretende Skalen, z.B. die Ganztonleiter. Ausgeschlossen werden müssen die in dieser Menge enthaltenen Transpositionen, also Skalen mit gleichen Intervallverhältnissen ab ces, ab c oder ab cis. Dabei ist zu beachten, dass zwei Stufen durch Alteration nicht nur deckungsgleich werden und sich damit aufheben, sondern sogar einander überkreuzen können (vgl. die Stufenfolge dis-eis-fes). 
entsprechend der unterschiedlichen Reihenfolge, in der \#- und b-Vorzeichen in der Generalvorzeichnung auftreten (Bsp. 1).

Eine zweite Nomenklatur bezeichnet das Ergebnis des Alterationsprozesses: Am einfachsten geschieht dies, indem die Skala mit ihren Tonhöhennamen aufgeschrieben wird, z.B. Phrygisch: c-des-es-f-g-as-b. (Alternativ könnten die Stufen bzw. Skalenpositionen mit den entsprechenden Vorzeichen einzeln oder zusammenfassend gekennzeichnet werden: 1 h, $2 b, 3 b, 4 h, 5$ h $, 6 b, 7 b$ oder auch b2367 1445 . Die ohne Punkt aufeinander folgenden Zahlen bezeichnen hier die Stufen.)

Ein weiteres Beispiel (Bsp. 3): ‘Zigeuner-Molk c-d-es-fis-g-as-h. Zunächst muss die Skala von $c$ aus geschrieben werden, weil dies von ces aus nur auf weniger seinfache Weise, mit Verdopplung einer Tonstufe (ces-cis-d... bzw. ces-des-d...) oder Doppelalteration (ces-des-eses...) möglich wäre. Diese Skala ist eine Skala zweiten Grades, eine additive

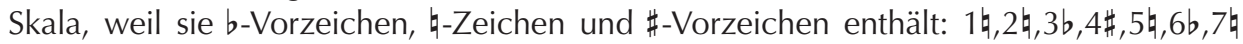
(bzw. b36月1257\#4), entstanden durch den Alterierungsprozess b1.4.5.6.7. \#1.

\section{Reduzierte Skalen}

Wir haben bis hierher eine Systematik diatonischer Skalen erhalten, die Bekanntes wie Dur, Moll, Kirchentonarten, Pentatonik, Ganztonleiter, Skalen mit übermäßigen Sekunden wie die senigmatische Skala soder bisher unbenannte Skalen umfasst. Andere Skalen wie z.B. die sBluestonleitern s sind bisher aber nicht in der Darstellung aufgetaucht, und es stellt sich dadurch die Frage, wie sie sich zu dem bisher entwickelten System verhalten. Wie kann das System weiter differenziert werden, um eine Klassifikation auch dieser Skalen zu ermöglichen?

Einen Anhaltspunkt zur Beantwortung dieser Frage bieten diejenigen subtraktiven Skalen, bei denen aufgrund der Alteration Töne weggefallen sind, weil sie sich in der gleichstufigen Stimmung klanglich mit einem benachbarten Ton vereinigten, etwa e mit $f e s, h$ mit ces. Diese Vereinigung der Töne führt zu einer Reduktion der Skalenstufenzahl $^{67}$, weshalb die entsprechenden Skalen reduzierte Skalen genannt werden sollen (Bsp. 4). Doch reduzierte Skalen gibt es nun nicht nur unter den subtraktiven, sondern auch unter den additiven Skalen. Hier ist z.B. die sPlaneten-Skalaı c-des-f-g-as zu nennen: Es ist eine hemitonische pentatonische Skala, die von Urmas Sisask so benannt wurde $^{68}$, aber bereits in der Antike ${ }^{69}$, in der isländischen ${ }^{70}$ und in der japanischen Musik (als »kumayoshi $\aleph^{71}$ ) bekannt ist. Sie kann in der Systematik nicht von ces aus, aber von c

67 Genaugenommen gilt dies nur auf der klanglichen Ebene; ich akzeptiere an dieser Stelle in der Begrifflichkeit aber die Ungenauigkeit, die nicht zwischen klanglicher und notierter Skala unterscheidet. Vgl. auch die Begriffe »Extentionalität« und »Intentionalität« von Tonmengen bei Rambold 2012, 156.

68 Vgl. Sisask 2005.

69 sskala des Olymposı e-f-a-h-c (vgl. Pöhlmann 1995, 1655).

70 Vgl. Annewanter 1977, 19. Annewanter erklärt die tonalen Gegebenheiten der von ihm untersuchten isländischen Zwiegesänge mit der Pentatonik e-f-a-h-c.

71 Sisask 2005, 3. Weiterführend zur Skalenbildung in der japanischen Musik vgl. Komoda/Mihoko 2002, 566-570. 
und cis aus notiert werden (da von ces aus eine Tonstufe verdoppelt oder Doppelalteration angewandt werden müsste, was weniger seinfach tik nicht präferierte Verfahren wäre). Sie stimmt mit fünf Tönen der phrygischen Skala, einer heptatonischen subtraktiven Skala, überein. Die zwei wegfallenden Töne lassen sich nicht allein durch das subtraktive Verfahren eliminieren, sondern für ihren Wegfall bedarf es zusätzlich eines additiven Schritts: e und $h$ müssen aufwärts alteriert werden. Die resultierende Skala lautet c-des-[eis]-f-g-as-[his]; eis und his entfallen. Die >PlanetenSkalas ist folglich innerhalb unseres Systems eine additive Skala mit eliminierten Tönen und kann daher als reduzierte diatonische Skala additiven Ursprungs verstanden werden.

Ein ähnliches Beispiel ist die hexatonische Skala ‘Blues-Mollı, normalerweise dargestellt als $c$-es-f-fis-g- $b$; sie lässt sich ebenfalls als reduzierte Skala additiven Ursprungs verstehen: $c$-dis-eis-fis-g-[ais]-b - entstanden durch den Alterationsprozess b2.3.4.5.6.7. \#1.4.5.6.

Als weiteres Beispiel einer reduzierten Skala sei die >Do-Pentatonikı $c-d$-e- $g$-a erwähnt. ${ }^{72}$ Ihre fünf Töne finden sich in mehreren subtraktiven Skalen, z.B. in Dur oder in der durch den Prozess b2.3.4.5.6. entstandenen Skala $c-d$-[e]-fes-g-a-b. ${ }^{73}$ Die $>$ DoPentatonikı ist in diesem Sinn ımehrdeutig und es fehlen ihr jene Töne (fes und his), die durch Halb- und Ganzton-Alteration eliminiert wurden. Außerdem lässt sie sich in einer zweiten Form über ces darstellen: ces-des-es-[fis]-ges-as-[h]. In beiden Formen handelt es sich um eine additive Skala mit eliminierten Tönen, aber die zweite Form ist gemäß der oben aufgestellten Regel ‘einfacher`(d.h. durch weniger Operationen von der Grundskala Ces-Dur ausgehend erreichbar); ihr gebührt daher der Vorzug.

Innerhalb der Tradition unseres Notationssystems gibt es nun entgegen den Gegebenheiten der Pedalharfe auch noch die Möglichkeit zur Doppelvorzeichnung. Diese soll daher zur Erklärung der Reduktion einer Skala in Betracht gezogen werden: Eine Doppelvorzeichnung mit der Konsequenz, dass ein Skalenton wegfällt, soll `Tilgung, einer diatonischen Tonstufe genannt werden. Skalen mit getilgten Tönen sind damit auch reduzierte Skalen, z.B. $c-d-e-f-(g=a s a s)-h$ oder $c$ - $($ disis $=e)-f-(g=a s a s)-h-c$. Auch die sPlaneten-Skala $a b$ cis müsste mit Doppelvorzeichnung geschrieben werden: $c i s-d$ [eses]-fis-gis-a-[heses]; die oben dargebotene Version c-des-[eis]-f-g-as-[his] ist aber im genannten Sinne seinfacher und soll daher gelten. Vorzeichenkombinationen, die die Doppelvorzeichnung übersteigen (also drei- und mehrfache \#- bzw. b-Vorzeichnungen) sollen aus der hier präsentierten Systematik ausgeschlossen sein.

Wir finden unter den reduzierten Skalen somit: subtraktive Skalen mit eliminierten Tönen, additive Skalen mit eliminierten Tönen oder Skalen mit per Doppelvorzeichnung

72 Die anhemitonischen pentatonischen Skalen werden mit den Solmisationssilben ihres untersten Tons bezeichnet, ausgehend von c-d-e-g-a, also z.B. 'So-Pentatonikı: g-a-c-d-e bzw. so-la-do-re-mi (vgl. Krüger 2011, 47-50; Bartók 1976, 364; Liang 1985, 58). Zur relativen Solmisation vgl. Heygster/ Grunenberg 1998.

73 Die Fragen, ob e oder fes in der Skala existiert, welcher der beiden Töne wegfällt, ob sie sich vereinigen oder ein Ton den anderen ersetzt, sind in der Gleichstufigkeit und bei enharmonischer Verwechslung, wenn unabhängig von tonalen Zusammenhängen argumentiert wird, letztendlich unerheblich. Welcher Ton eingeklammert wird, muss der Zusammenhang ergeben. Hier wurde in einem materialen, nicht auf eine bestimmte Musik bezogenen Kontext derjenige Ton eingeklammert, der in höherem Maß alteriert worden ist. 
getilgten Tönen. Reduzierte Skalen könnten daher als Unterklasse sowohl der subtraktiven als auch der additiven Skalen angesehen werden, bilden aber als sreduzierte eigene dritte Kategorie, die Skalen dritten Grades. Innerhalb der Klasse der reduzierten Skalen können dann die pentatonischen von den hexatonischen, die Skalen subtraktiven von jenen additiven Ursprungs und Skalen mit eliminierten von jenen mit getilgten Tönen unterschieden werden. Unten wird sich zeigen, dass auch erweiterte Skalen mit weniger als sieben Tönen hier eingruppiert werden, wodurch reduzierte Skalen allgemein als Klasse von Skalen mit weniger als sieben Tönen definiert werden können (siehe Abschnitt Erweiterte Skalen). Eine Folge dieser Bestimmung von reduzierten Skalen ist, dass die Klassen der subtraktiven, additiven und der unten zu beschreibenden erweiterten Skalen umgekehrt zu Klassen heptatonischer Skalen werden.

Klar ist auch, dass heptatonische Skalen - als gebrochene Cluster ${ }^{74}$ verstanden grundsätzlich sieben Mal reduziert werden können. So entstehen neben hexa- und pentatonischen Skalen durch das subtraktiv-additive Verfahren auch tetratonische Skalen, durch Doppelvorzeichnung zudem tritonische Skalen (klanglich gebrochenen Drei- und Vierklängen entsprechend, vgl. z.B. die Skala ces-d-[e]-fes-[gis]-as-[h]). Eine weitere Reduktion ist danach nur noch durch Streichung von Skalenstufen möglich, sodass zunächst ein Intervall, dann ein Ton und schließlich kein Ton mehr steht (aus einer Skala wird eine Form von Nichts): Kategorien, die zwar prinzipiell noch als Skalen aufgefasst werden könnten, aber, da sie selbst Bausteine von Skalen darstellen, aus logischen Gründen vom Skalenbegriff ausgeschlossen bleiben sollen.

Die reduzierten Skalen lassen sich in Entsprechung zum Gesagten wie folgt unterteilen:

- Tritonische Skalen / gebrochene Dreiklänge

- Tetratonische Skalen / gebrochene Vierklänge

- Pentatonische Skalen

a) subtraktiven Ursprungs

b) additiven Ursprungs

c) erweiterten Ursprungs (siehe unten)

- mit getilgten Tönen (Doppelvorzeichnung)

- mit gestrichenen und hinzugefügten Tönen

- Hexatonische Skalen

a) additiven Ursprungs

b) erweiterten Ursprungs (siehe unten)

- mit getilgten Tönen (Doppelvorzeichnung)

- mit gestrichenen und hinzugefügten Tönen

74 Ich verstehe hier `Cluster unter der Prämisse der Gleichstufigkeit als `Tonhaufen` oder »Tontraube« (Eimert/Humpert 1973, 49; vgl. auch Cowell 1996, 117-121; Schneider 1975, 231). Unklar ist im Deutschen, welches Genus der Begriff hat, Maskulinum oder Neutrum. Deutlich werden an dieser Stelle Übersetzungsprobleme, die als eigenständige Schwierigkeit der Musiktheorie thematisiert werden sollten. 
An dieser Stelle ist nochmals festzuhalten, dass Elimination und Tilgung das Wegfallen von Tönen aufgrund von Alteration bewirken. Zunächst implizit, dann per definitionem (`reduzierte Skalen`) vollzog die vorliegende Argumentation einen Übergang zu Operationen auf der Ebene der Anzahl von Skalenstufen. Das Potenzial des Vorgehens, Vorzeichen wegzustreichen oder hinzuzufügen, ist im Rahmen des hier präsentierten Systems nunmehr ausgeschöpft. Dies führt zur nächsten Skalenklasse, den erweiterten Skalen.

Pentatonische Skala subtraktiven Ursprungs (mit eliminierten Tönen)

b1. 2 . So-Pentatonik / chin. Modus 4 Zhǐ / Gilchrist Mode 1

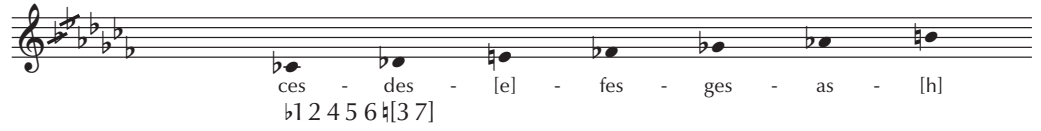

Pentatonische Skalen additiven Ursprungs (mit eliminierten Tönen)

b1. 2. 5. 6.7. \#6.7. Planeten-Skala / kumayoshi / Olympos-Skala

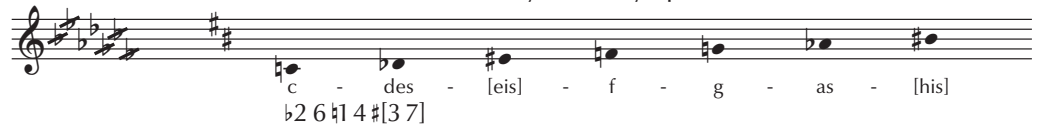

b1. 2. 3. 4. 5. 6. $\sharp 7$. Do-Pentatonik / chin. Modus 1 Gong / Gilchrist Mode 3

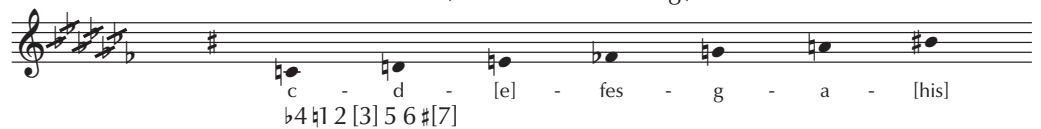

b1. 2. 3. 5. 6. $\sharp 7 . \quad$ Alterierte Do-Pentatonik (hemitonische Pentatonik)

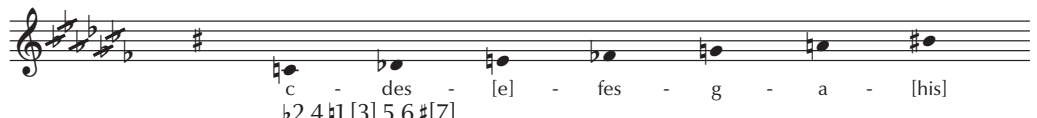

Pentatonische Skalen, durch Tilgung entstanden b1. 4. 5. 6. 7. \#1.7. 判1.

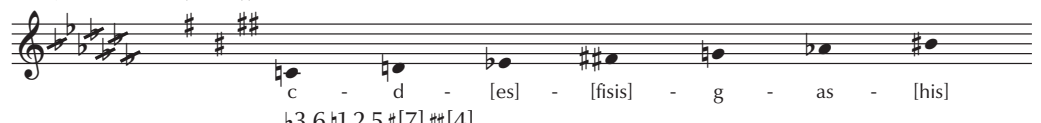

b1. 4.5.6.7. b2. 3 .

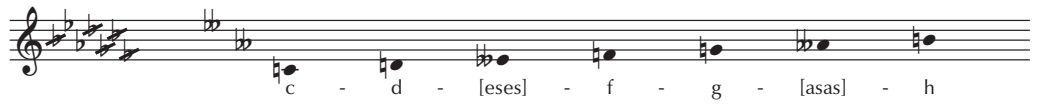

bb[3 6] 61 2457

b1. 2. 4. 5. 6. 7. $\sharp 4.6 b 3 . \sharp \sharp 4$.

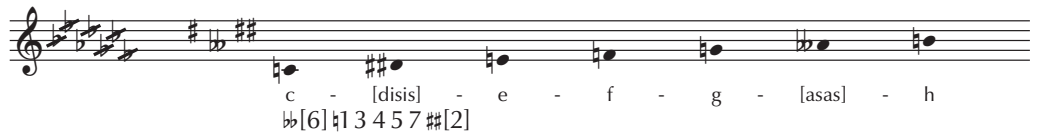

Beispiel 4: Reduzierte Skalen (Auswahl) ${ }^{75}$

75 Der optischen Anschaulichkeit halber verwendet die hier entwickelte Systematik, in Analogie zum Vorzeichen >Doppel-bb<, die Kombination \#\# anstelle des herkömmlichen Doppelkreuzes. 
Hexatonische Skala subtraktiven Ursprungs

b1.

Gilchrist Mode 1A / 3B

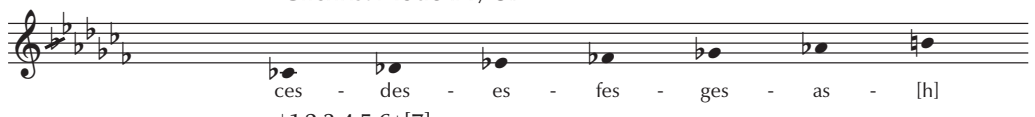

b1 23456 ต[7]

Hexatonische Skalen additiven Ursprungs

Übermäßige Skala (1. Version) / Faustmodus / Halbton-Kleinterz /

b1 2. 3. 5 6. 7. \#3. 7. 8. Modus mit begrenzter Transpositionsmöglichkeit (Schuster-Craig) / Alternierende Sechsstufigkeit

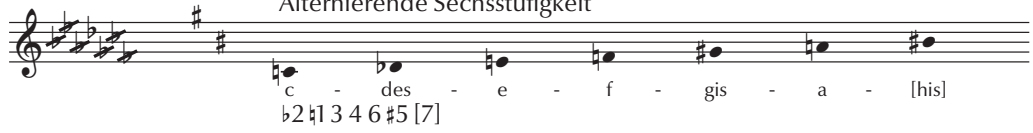

b1. 2. 3. 4. 5. 6. \#4. Übermäßige Skala (2. Version) / Faustmodus / Kleinterz-Halbton / ...

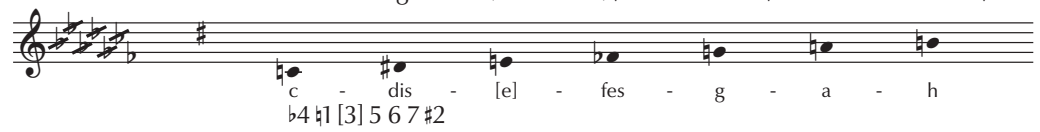

Hexatonische Skala, durch Tilgung entstanden

b1. 2. 4. 5. 6. 7.bb3.

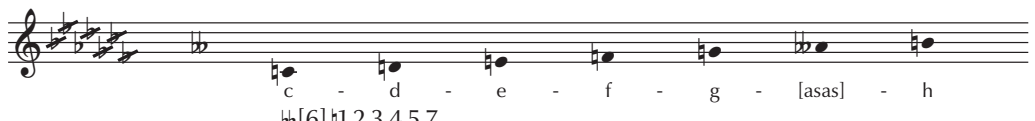

bb[6] 6123457

Beispiel 4 (Fortsetzung)

\section{Erweiterte Skalen und reduzierte Skalen erweiterten Ursprungs}

Es gibt nun Skalen, die zwar aus sieben Tönen bestehen, sich jedoch nicht allein durch das subtraktiv-additive Verfahren, sondern nur mithilfe von Doppelvorzeichnung(en) oder durch Streichen und Hinzufügung von Tönen bilden lassen - sie sollen deshalb serweiterte Skalen` genannt werden. `Blues-Dur`, häufig angegeben als c-es-e-f-fis-g- $b$, ist solch ein Fall (Bsp. 5). ${ }^{76}$ Es lässt sich auch darstellen als c-dis-e-f-ges-asas-b. Die Töne $c$, e, $f$ können subtraktiv erklärt werden; additiv kommt der Ton dis zustande; ges und $b$ sind noch Teil der Stammtonskala von Ces-Dur; der Ton $g$ muss als asas durch Doppelvorzeichnung gebildet werden. Es gibt also heptatonische Skalen, in denen Töne, die andernfalls nur als hinzugefügte zweite Form einer bereits vorhandenen Skalenstufe erscheinen könnten, durch enharmonische Verwechslung und Doppelvorzeichnung gebildet werden müssen. Zu solchen erweiterten Skalen zählen auch `Harmonisch-Moll` $\left(\mathrm{HM}^{7}\right)$ c-des-es-fes-ges-as-a und `Harmonisch-Dur`( $\left.\mathrm{HD}^{7}\right)$ c-des-es-f-ges-as-a: Scheinbar ist das $h$ in beiden Fällen weggefallen und a hinzugefügt worden. Erklärbar ist dies im Rahmen der hier vorgestellten Systematik dadurch, dass ein Ton durch Doppelvorzeich-

76 Weil die Blue Notes nicht eindeutig einer Tonhöhe innerhalb des halbtönig-gleichstufigen Tonsystems zugeordnet werden können, lassen sie sich genaugenommen auch nicht in die vorliegende Systematik einordnen. Da jedoch `Blues-Dur und ‘Blues-Molk auch in der Gleichstufigkeit unter diesen Namen benutzt werden, werden sie hier besprochen. 
nung und enharmonische Verwechslung zu einer zweiten Form seines Nachbarn wurde (heses wurde zu a).

An dieser Stelle wird klar, dass von ces aus eine Doppelvorzeichnung mit \#-Zeichen als dritte ^Halbtontransposition« nach oben erscheint (ces-c-cis-cisis), eine Vorzeichnung mit Doppel-b aber lediglich als `Halbtontransposition` nach unten (ces-ceses). Allgemein gesprochen hat also eine Doppelvorzeichnung in unserem Zusammenhang die Konsequenz, dass entweder eine Tonstufe entfällt (Tilgung) oder eine doppelt vorhandene entsteht (Ergänzung). Doppelvorzeichnung soll deshalb letztlich dem Bereich der erweiterten Skalen zugeordnet werden. Skalen, die mithilfe von Doppelvorzeichnung entstehen, sollen als (siebentönige) serweiterte Skalen bildete) `reduzierte Skalen erweiterten Ursprungs` bezeichnet werden. Doppelvorzeichnung soll grundsätzlich als weniger seinfach gelten im Vergleich zu subtraktiv-additivem Vorgehen, aber als seinfacher im Vergleich zu bloßer Streichung oder Hinzufügung von Tönen.

Es gibt nun auch solche heptatonische Skalen, die nicht mittels Doppelvorzeichen, sondern nur durch Streichung und Hinzufügung von Tönen gebildet werden können. Dies sei am Beispiel der Skala $c^{-}+$cis- $d$-+dis-e-f-ges-[as]-[his] erläutert. ${ }^{77}$ Hier sind die Töne $c, d, e, f$ subtraktiv erklärbar, ges gehört noch zur Stammtonskala von Ces-Dur, his ist additiv erklärbar, cis und dis aber sind hinzugefügt - sie sind nicht durch Doppelvorzeichnung konstruierbar, sie zeigen vielmehr einen jeweils zweiten Alterationszustand eines bereits in der Skala enthaltenen Stammtons. Auch der Wegfall des Stammtons as ist weder durch Subtraktion, Addition noch durch Doppelvorzeichnung erklärbar.

Generell gesprochen heißt das: Es gibt Skalen diatonischer Herkunft, denen für jeden weggefallenen Ton ein neuer Ton hinzugefügt worden ist (ich verwende den Begriff ıweggefallen` als Oberbegriff für seliminiertı, sgetilgt‘, sgestrichen`). Die erweiterten Skalen stehen damit erstens in der Nähe der transheptatonischen Skalen, die durch mehr als sieben Töne gekennzeichnet sind, verbleiben aber im Gegensatz zu diesen innerhalb der Heptatonik. Sie sind zweitens durch die Dopplung von Tonstufen mit der chromatischen Skala verwandt.

Bezüglich ihrer Anzahl können erweiterte Skalen als diejenigen heptatonischen Skalen betrachtet werden, die der Differenz zwischen den 792 aus den zwölf Tonhöhen des chromatischen Totals konstruierbaren Siebentongruppen und den 359 subtraktiven und additiven Skalen $(792-359=433)$ entsprechen.

Als Beispiel für eine serweiterte Skala mit weniger als sieben Tönen (reduzierte Skala erweiterten Ursprungs) sei noch $c-+c i s-d$-+dis-e-f-[geses]-[as]-[his] erwähnt. Es ist die eben besprochene clusterartige heptatonische Skala ohne ges. Durch Doppelvorzeichnung vereinigen sich hier zusätzlich $f$ und geses, und es entsteht eine hexatonische Skala. Folglich wird unter den reduzierten Skalen eine weitere, oben bereits angemerkte Unterklasse erkennbar: die erweiterten Skalen mit weniger als sieben Tönen. Um die Systematik bzw. Nomenklatur einfach zu halten, sollen nun alle Skalen mit weniger als sieben Tönen ıreduzierte Skalen` genannt werden, auch dann, wenn sie hinzugefügte Töne

77 Mit + wird ein zusätzlicher, mit [ ] ein weggefallener Ton bezeichnet; zur Nomenklatur siehe unten, Abschnitt Nomenklatur 2. 
enthalten. ${ }^{78}$ Zum entscheidenden Charakteristikum der Klasse der reduzierten Skalen werden damit nicht Operationen auf der Ebene der Alteration wie Subtraktion, Addition, Elimination oder Tilgung, und auch nicht Erweiterung, sondern die geringe Tonanzahl erklärt, obwohl die reduzierten Skalen natürlich von den genannten Operationen geprägt sind. Die reduzierten Skalen, auch ıSkalen dritten Grades`, werden dadurch zu einem Sammelbecken von Skalen unterschiedlichster Art.

Erweiterte Skalen sind damit nun als heptatonische Skalen mit weggefallenen und hinzugefügten Tönen die vierte Skalenklasse, weshalb sie ıSkalen vierten Grades` heißen sollen. Weil aber hinzugefügte Töne auch im Bereich der transheptatonischen Skalen eine zentrale Rolle spielen, ist spätestens mit den erweiterten Skalen ein Grenzbereich der diatonischen Skalen und damit eine Grenze der Leistungsfähigkeit der hier vorgestellten Systematik erreicht. ${ }^{79}$

\section{Nomenklatur 2: Aspekte der reduzierten und erweiterten Skalen}

Die bereits vorgestellte Nomenklatur muss nun ergänzt werden: Weggefallene Töne werden durch eine eckige Klammer gekennzeichnet, hinzugefügte Töne durch ein + (die zugehörigen Notenköpfe sind in den Notenbeispielen durch ein Quadrat umrahmt). sBlues-Molk kann prozesshaft folgendermaßen dargestellt werden: b3.4.5.6.7.\#4.5.+fis; das Ergebnis ist die Skala: $c$ - [dis]-es-f-fis-g-[ais]-b. bzw. 1h, [2甘],3b,4h, $+4 \sharp, 5$, $,[6 \sharp], 7 b$.

Ein zweites Beispiel: Die sSo-Pentatonikı ces-des-[e]-fes-ges-as-[h] muss von ces aus geschrieben werden, weil die Schreibweise $c$ - $d$-f-g-a nur additiv möglich wäre (mit eis) und damit weniger seinfach te Skala, die durch den Alterierungsprozess b1.2. entsteht; das Ergebnis lässt sich mit $1 b, 2 b,\left[3 h^{2}\right], 4 b, 5 b, 6 b,\left[7 h^{-}\right]$darstellen.

Im Hinblick auf die Nomenklatur bedeutet das: In der Notation subtraktiver Skalen (Skalen ersten Grades) treten $b$-Vorzeichen und $\$$-Zeichen auf, bei additiven Skalen (Skalen zweiten Grades) zudem \#-Vorzeichen; reduzierte Skalen (Skalen dritten Grades) haben weniger als sieben Töne, sodass in jedem Fall eckige Klammern [ ] auftreten; die erweiterten Skalen (Skalen vierten Grades) enthalten wiederum sieben Töne, und zu allen bisher genannten Zeichen sind auch + (Pluszeichen) oder Doppelvorzeichen ( $b b$, \#\#) nötig. ${ }^{80}$

Damit eine Skala analytisch in die Systematik eingeordnet werden kann, muss sie zunächst, wenn möglich, auf ces notiert werden. Wenn dies nicht gelingt, weil dabei eine

78 Die alternative Möglichkeit, erweiterte Skalen mit weniger als sieben Tönen den erweiterten Skalen zuzurechnen, schüfe die Uneindeutigkeit, dass sowohl in der Klasse der reduzierten Skalen als auch in der der erweiterten Skalen mit weniger als sieben Tönen enthalten wären.

79 Die Unterteilung der Skalen in tetratonisch, pentatonisch, hexatonisch, heptatonisch würde auch eine rein an der Anzahl der Töne orientierte Skalenklassifikation erlauben. Dies könnte insgesamt zu einer Klassifikation von Skalen in subheptatonisch, heptatonisch und transheptatonisch führen. Die getroffenen Unterscheidungen subtraktiv, additiv und erweitert würden es dann ermöglichen, die heptatonischen Skalen zu differenzieren.

80 Der optischen Anschaulichkeit halber verwendet die hier entwickelte Systematik, in Analogie zum Vorzeichen >Doppel-b`, die Kombination \#\# anstelle des herkömmlichen Doppelkreuzes. 
b2. 4. 6.7. $\$ 4.6 b 3$.

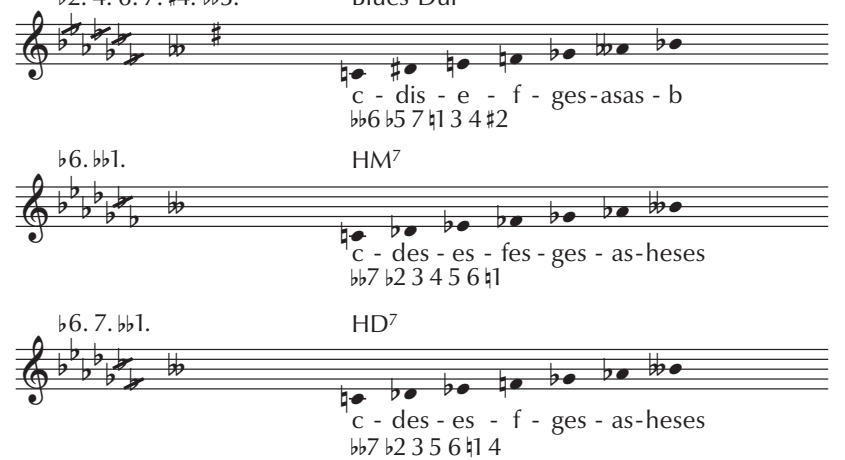

b1. 2. 4. 6. 7. \#7. - as + cis dis

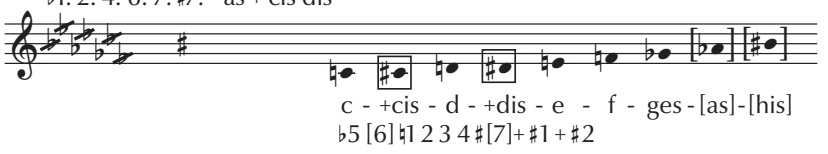

b1. 2. 4. 6. 7. $\sharp 7 . b 65$. - as + cis dis
Beispiel 5: Erweiterte Skalen (Auswahl)

Tonstufe doppelt verwendet werden müsste, muss derselbe Vorgang auf c oder dann auch auf cis versucht werden. So können nach und nach alle vier Skalengrade durchgearbeitet werden bis die bestmögliche - sprich: im System ıeinfachste - Notationsform gefunden wird und dadurch die Skala eingruppiert werden kann.

\section{GRENZEN DES SYSTEMS}

Schon bei den reduzierten Skalen wurde deutlich, dass dieses System wie jeder Klassifizierungsversuch an Grenzen stößt: Vor allem Mehrdeutigkeiten und Klassifizierungsalternativen fordern Entscheidungen heraus, die offengelegt und argumentativ abgesichert werden müssen. Kritisch ist außerdem an dieser Stelle einzuwenden, dass bei den reduzierten und erweiterten Skalen das Alterationsverfahren, wie es bei den ersten beiden Skalenklassen genutzt worden war, verlassen wurde - eben weil es an seine Grenze gekommen war - und ein ambivalentes Verfahren angewandt wurde, das einerseits zwar noch Alterationen von Stufen nutzt, andererseits aber faktisch die Zahl der Stufen verringert oder vermehrt, wie es die Begriffe ıreduziert und serweitert suggerieren. Aus 
diesen Gründen ist es nun notwendig, die Grenzen des Systems näher zu betrachten, darzustellen und auszuloten. Dies geschieht im Folgenden durch die Beschreibung der Eigenheiten wichtiger Skalengruppen, die, anders als in üblicher Darstellung, von der hier entwickelten Systematik nicht als homogene Gruppen erfasst werden können. Dadurch gelingt eine Klärung und Differenzierung des bisher Entwickelten.

\section{Modi mit begrenzter Transponierbarkeit}

Durch die erweiterten Skalen wurde die Grenze der diatonischen Skalen erreicht, weil laut obiger Definition eine Erweiterung einer diatonisch-heptatonischen Skala streng genommen eine transheptatonische Skala schafft - eine Skala mit mehr als sieben Tönen. Die »Modi mit begrenzter Transpositionsmöglichkeit « ${ }^{81}$ von Olivier Messiaen - das sind ssymmetrische Skalen`, die durch drehsymmetrische Figuren im Quintenzirkel gekennzeichnet sind, was ihre beschränkte Transponierbarkeit anschaulich macht (Bsp. 6) ${ }^{82}$ bestimmen nun diese Grenze näher als ein Modi- bzw. Skalen-Feld (Bsp. 7), das gemäß der Nomenklatur des vorliegenden Systems sowohl diatonisch-heptatonische als auch transheptatonische Skalen enthält: Messiaen stellt eine reduzierte Skala subtraktiven Ursprungs (die Ganztonleiter ces-des-es-f-g-a-h, entstanden durch die Alteration b1.3.5.7.; bei Messiaen der 1. Modus) mit einer reduzierten Skala mit getilgtem Ton - Doppelvorzeichnung, daher reduzierte Skala erweiterten Ursprungs - (5. Modus bei Messiaen) und fünf transheptatonischen Skalen aufgrund der gemeinsamen Eigenschaft der begrenzten Transponierbarkeit als eine Gruppe von `Modi< zusammen. Als solche sprengt sie aber die Gruppierungs- und Klassifizierungsregeln der hier vorgestellten Systematik und weist dadurch auf deren Grenzen hin.

Messiaens System erfasst freilich nicht alle Skalen begrenzter Transpositionsmöglichkeit. Zu nennen wäre etwa der von John Schuster-Craig beschriebene »8. Modus mit begrenzter Transpositionsmöglichkeit « ${ }^{83}$. Es handelt sich um die reduzierte Skala additiven Ursprungs ces-d-es-fis-g-[ais]-b (Kleinterz-Halbton-Skala) bzw. c-des-e-f-gis-a-[his] (Halbton-Kleinterz-Skala). ${ }^{84}$

81 Messiaen 1966, 56. Vgl. Messiaen 2012, 458-476.

82 "Solche Skalen werden [auch] als ıIntervallzyklen` bezeichnet, bei denen die Oktave durch ein Intervall bzw. durch eine Kombination mehrerer Intervalle gleichmäßig unterteilt wird « (Kuhn 2014, 252; Ergänzung des Verfassers). Deshalb sind genaugenommen auch die chromatische Skala, der verminderte Dreiklang, der übermäßige Dreiklang und der Tritonus sbegrenzt transponierbarı. (Im Deutschen werden von Übersetzer*innen und Autor*innen die Begriffe sbegrenzte Transponierbarkeit und sbegrenzte Transpositionsmöglichkeit synonym verwendet.) Zu Symmetrien in der Musik allgemein, insbesondere zum »Achsensystem« bei Bartók vgl. Lendvai 1957, 91-103.

83 Vgl. Schuster-Craig 1990 (»An Eighth Mode of Limited Transposition«). Diese Skala ist identisch mit dem sogenannten »Faust-Modus« (Krüger 2011, 119), so benannt, weil er von Franz Liszt in seiner Faust-Symphonie eingesetzt wurde (vgl. auch Schuster-Craig 1990, 300f.), bzw. der »alternierenden Sechsstufigkeit« (Kissenbeck 2007, 110), auch bekannt als ıübermäßige Skala` (so benannt, weil sich aus ihren Skalentönen zwei übermäßige Akkorde bilden lassen; vgl. Haunschild 1992, 140ff.).

84 Vgl. Kissenbeck 2007, 110 (dort abgekürzt als »KTe-HT« bzw. »HT-KTe«). Diese in der Jazztheorie gängigen Bezeichnungen orientieren sich an den in der Skala vorkommenden Intervallverhältnissen. In gleicher Weise benannt ist die »alternierende Achtstufigkeit« (ebd., 109): Ganzton-Halbton-Skala 
Insgesamt charakterisieren die `Modi mit begrenzter Transponierbarkeit den Bereich zwischen den Skalenklassen der hier vorgestellten Systematik sowie zwischen den Merkmalen diatonisch und chromatisch als ein Übergangsfeld, auf dem Messiaen gezielt spolymodak komponierte. ${ }^{85}$

Begrenzte Transpositionsmöglichkeit (am Beispiel der Ganzton-Halbton-Skala)

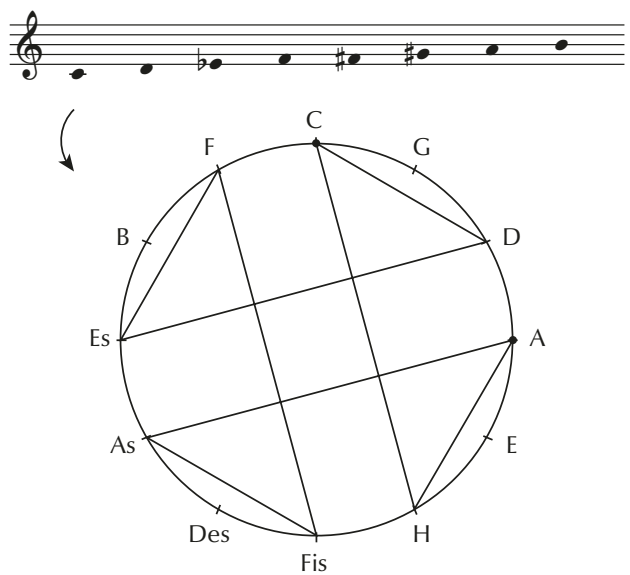

$\Rightarrow 3$ Transpositionsmöglichkeiten

Beispiel 6: Drehsymmetrie am Beispiel der Ganzton-Halbton-Skala

\section{Anhemitonische Pentatonik}

Eine andere Grenze des hier vorgestellten Systems zeigen die anhemitonischen pentatonischen Skalen auf. ${ }^{86}$ Die Skalen der , Re-, Mi-, So-, und La-Pentatonikı kommen in der vorliegenden Systematik als subtraktive Skalen mit eliminierten Tönen vor (reduzierte

(GT-HT) und Halbton-Ganzton-Skala (HT-GT), Messiaens 2. Modus. Mike Rossi (2007, 13) nennt die beiden Formen dieser »Diminished Scale« »Mode 1«(GT-HT) und »Mode 2« (HT-GT). Für weitere Literatur zur oktatonischen Skala vgl. Taruskin 1985 sowie Kahan 2009. »Die oktatonische Skala hat Bartók besonders interessiert, denn sie erlaubte ihm, zwischen diatonischen und chromatischen Tonräumen zu vermitteln« (Kuhn 2014, 253). Zu »Skalenbildung aus dem Wechsel zweier Intervalle« mithilfe der Fibonacci-Reihe vgl. ebd., 241.

85 Vgl. Messiaen 1966, 66ff. Anders bezeichnete Bartók in seinen Harvard Lectures »polymodale Chromatik« (Bartók) als »ein Verfahren, chromatisch ausgefüllte Tonräume durch Überlagerung diatonischer Skalen oder Skalenausschnitte zu schaffen« (Kuhn 2014, 256). Kuhn betont weiter, dass »Polymodalität [...] häufig durch symmetrische Spiegelstrukturen zustandekommt« (ebd., 257). »Anhand der diatonischen Skalen lässt sich zeigen, dass mit Ausnahme des Dorischen alle Skalen in der Spiegelung einen neuen Modus erzeugen« (ebd., 258). So wird z.B. Phrygisch aufwärts g-as- $b$ $c$ - $d$-es-f-g zu lonisch abwärts g-fis-e-d-c-h-a-g.

86 Siehe oben, Anm. 72. Vgl. Bartók 1976, 364. Die anhemitonische Pentatonik wird auch mit chinesischen Skalen assoziiert: "Gong (Do) mode«, "Jiao (Mi) mode«, "Zhi (sol) mode", "Yu (La) mode» (Liang 1985, 58; vgl. zu anderen chinesischen Skalen ebd., 66, 85, 92 und 111). Vgl. zur weiteren Differenzierung Chen 2002, 120-122. 
Messiaen: Modi mit begrenzter Transponierbarkeit

Ganztonleiter

b1. 3.5.7. Ganztonleiter

I

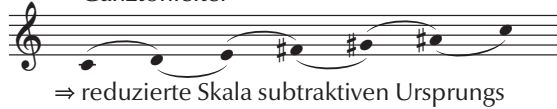

b.

$\Rightarrow$ reduzierte Skala subtraktiven Ursprungs

b1 23 4 56 [7]

II

HT-GT (alternierende Achtstufigkeit)

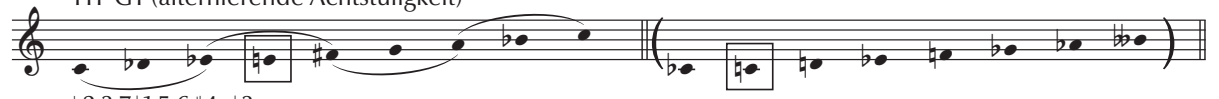

b2 37 1 $56 \# 4+$ 3

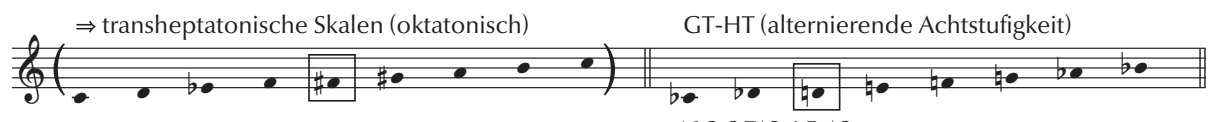

b1 267 औ $45+\natural 2$

III

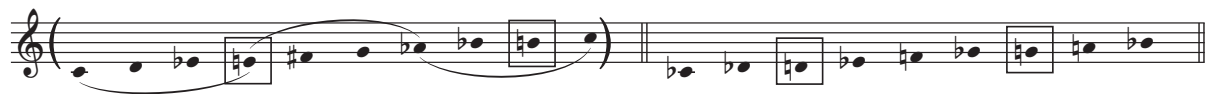

$\Rightarrow$ transheptatonische Skala (nonotonisch)

b1 2357 46+๓25
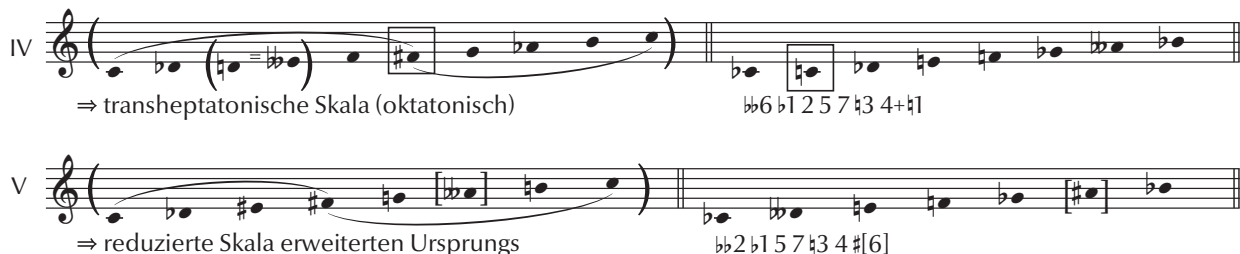

$\Rightarrow$ reduzierte Skala erweiterten Ursprungs $\quad$ bb 2 b1 57 \$ $4 \sharp[6]$
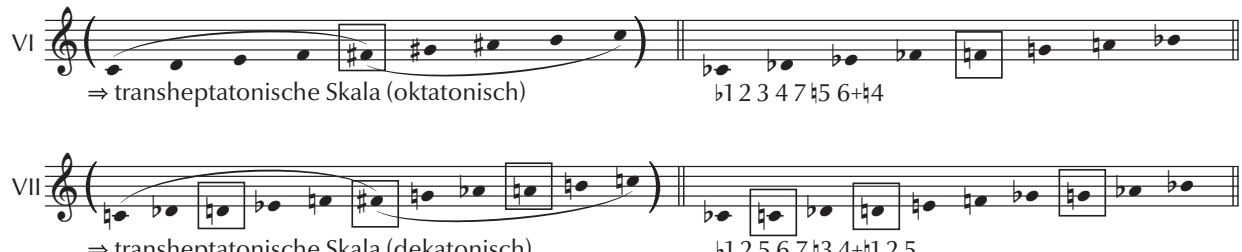
$\Rightarrow$ transheptatonische Skala (dekatonisch)
b1 2567 3 4+ฬ125

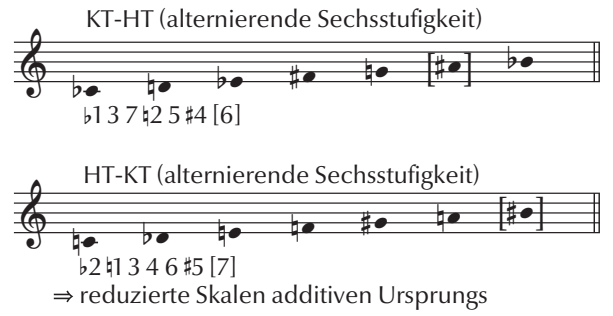

Beispiel 7: Symmetrische Skalen 
Skalen subtraktiven Ursprungs), sogar mit ähnlichen Zahlenreihen in ihrer Entstehung: b1.2.; b1.2.3.; b1.2.3.4.; b1.2.3.4.5.; es handelt sich also um kontinuierlich alterierte Skalen. Die sDo-Pentatonikı c- $d$-e-[fes]-g-a-[his] hingegen lässt sich nicht einfach durch subtraktive Alterationen erreichen, sondern nur mithilfe eines additiven Schritts: b1.2.3.4.5.6. \#7. oder seinfacher b1.7.\#1. (ces-des-es-[fis]-ges-as-[h]) (siehe oben, Abschnitt Reduzierte Skalen). Das heißt, die Zusammengehörigkeit der anhemitonisch pentatonischen Skalen als Gruppe kann mit der hier entwickelten Systematik nicht dargestellt werden. Diese Einschränkung kann allerdings kompensiert werden, indem die reduzierten Skalen, wie oben beschrieben, als eigene Klasse und nicht als Unterklassen der subtraktiven, additiven und erweiterten Skalen betrachtet werden. Auf diese Weise gehört jede Pentatonik zu den reduzierten Skalen, den Skalen dritten Grades.

\section{`Harmonisch-Molk (HM), , Melodisch-Molk (MM), ’Harmonisch-Dur` (HD)}

Ein ähnliches Problem - das Auseinanderbrechen einer üblicherweise als Einheit dargestellten Skalengruppe - taucht auch bei denjenigen >Modi auf, die Andreas Kissenbeck durch Rotation der Skalen `Harmonisch-Molk ( $\mathrm{HM}^{1}$ : $c-d$-es-f-g-as- $h$ ), ’Melodisch-Molk (MM': c- $d$-es-f-g-a- $h$ ) und `Harmonisch-Dur (HD': c- $d$-e-f-g-as- $h$ ) bestimmt - wobei er auch $\mathrm{HM}^{1}$, MM ${ }^{1}$ und $\mathrm{HD}^{1}$ selbst Modi« nennt (Bsp. 8, 9 und 10). ${ }^{87}$ Keine dieser ModusGruppen - im Sprachgebrauch des vorliegenden Aufsatzes: Skalengruppen - lässt sich als Ganze in einer der vier oben entwickelten Skalenklassen unterbringen. (Lediglich die im Jazz ebenfalls Verwendung findenden `Kirchentonarten sind sämtlich subtraktive Skalen - sie wären nach dem eben zitierten, hier nicht verwendeten Sprachgebrauch >Modi von lonisch, $\mathrm{I}^{1-7}$ ). Bei `Harmonisch-Molk z.B. bilden lediglich $\mathrm{HM}^{2}, \mathrm{HM}^{3}, \mathrm{HM}^{5}$ und $\mathrm{HM}^{6}$ eine Gruppe subtraktiver Skalen, $\mathrm{HM}^{4}$ ist eine additive Skala und $\mathrm{HM}^{7}$ eine erweiterte Skala. Das Auseinanderbrechen dieser Skalengruppe(n) ergibt sich, weil im hier entwickelten System ausgehend von der Ces-Dur-Skala jedem Ton zwar zwei ^Halbtonerhöhungen zugestanden werden, beide ıHalbtonerhöhungen aber je verschiedenen Skalenklassen zugeordnet wurden (subtraktiv und additiv). Außerdem wurde davon ausgegangen, dass, sobald eine dritte Alteration durch Doppel-b oder Doppel-\# notwendig ist, um eine Skala zu erklären, diese Skala dann in die Kategorie der erweiterten Skalen fällt bzw. bei geringerer Tonanzahl als sieben in die Klasse der reduzierten Skalen.

Das heißt, die Darstellung von Skalen durch Rotation und deren Klassifikation im hier vorgestellten, bei Alterationen seinen Ausgang nehmenden System, können oft nicht zur Deckung gebracht werden. In der Jazztheorie werden erstaunlicherweise aber bei der Benennung von Skalen beide Möglichkeiten kombiniert verwendet: Skalen werden dort

87 Vgl. Kissenbeck 2007, 101-111; vgl. ebd., 101: „Einbindung weiterer Skalen und deren Modi (Superimposition) «. Hingewiesen sei auf die unterschiedliche Zuordnung der Begriffe sModus« und `Skalas bei Idelsohn (siehe oben, Anm. 64) und Kissenbeck. Haunschild bezeichnet das, was Kissenbeck »Modi (Superimposition) « nennt, als »Stufenskalen« (Haunschild 1992, 136). Wenn man von Kissenbecks Zuordnung der Begriffe ‘Skalaı und `Modus` ausgeht und Skalen probehalber als Cluster betrachtet, dann würden deren `Modi Synonyma zu den `Lagen eines Clusters` (zum Clusterbegriff siehe oben, Anm. 74). 
einerseits mithilfe der Namen der 'Kirchentonarten` (gewonnen durch Rotationen der ionischen Skala) benannt, andererseits durch die Nennung der Alterationen (von Akkordund Optionstönen) näher bestimmt (z.B. HD ${ }^{5}=$ »Mixolydisch-b9 « mit 7/b9/11/13). ${ }^{88}$ Gerade diese kombinierende Bezeichnungsweise zeigt den aufgewiesenen Bruch zwischen den beiden möglichen Darstellungsformen von Skalen: mithilfe von Rotationen oder ausgehend von Alterationen (Bsp. 8, 9 und 10) ${ }^{89}$.
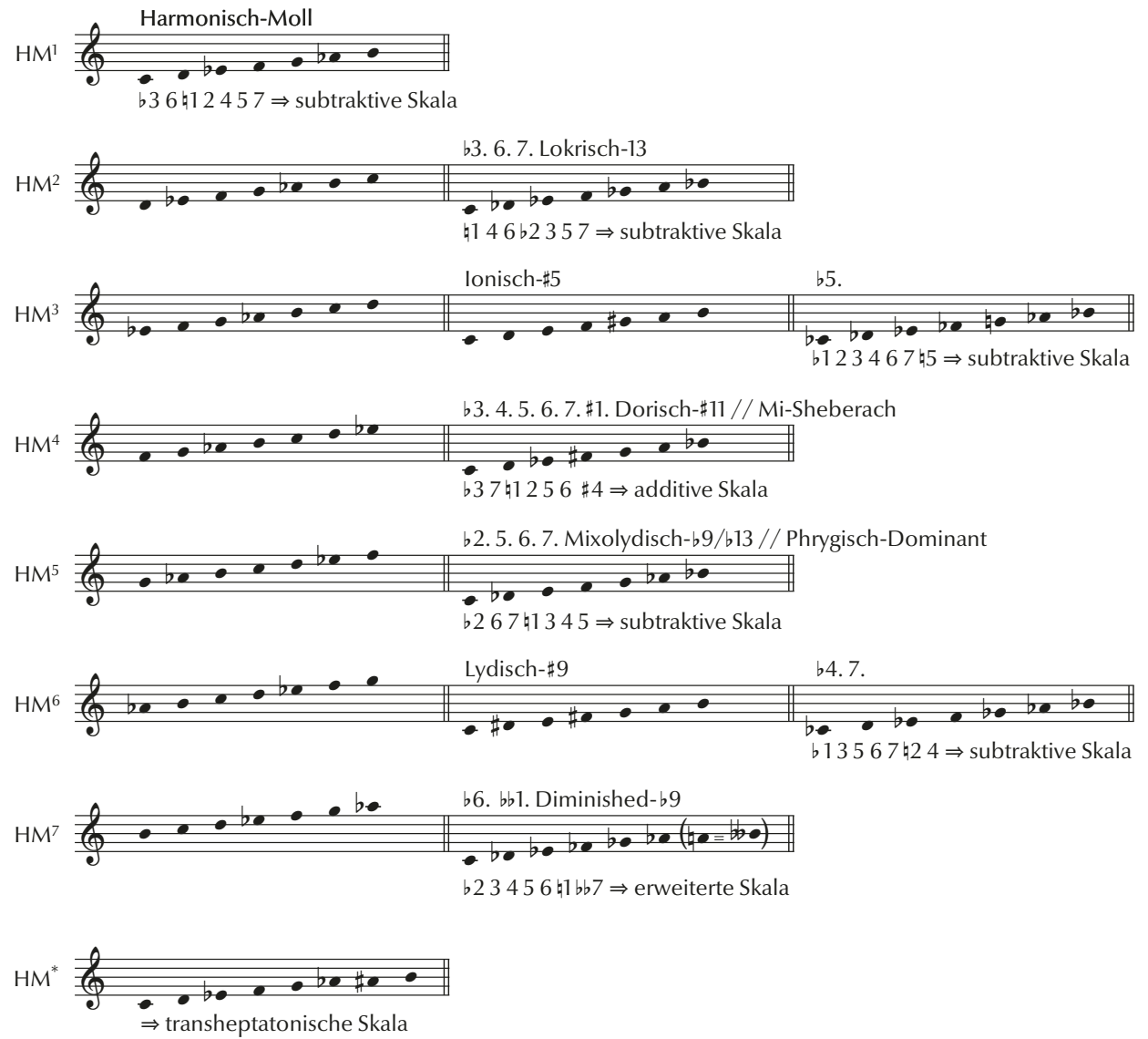

Beispiel 8: ^Harmonisch-Molk

88 Vgl. Kissenbeck 2007, 107.

89 Die an die Kirchentonarten angelehnten Bezeichnungen sind Kissenbeck 2007, 103-107, entnommen. 

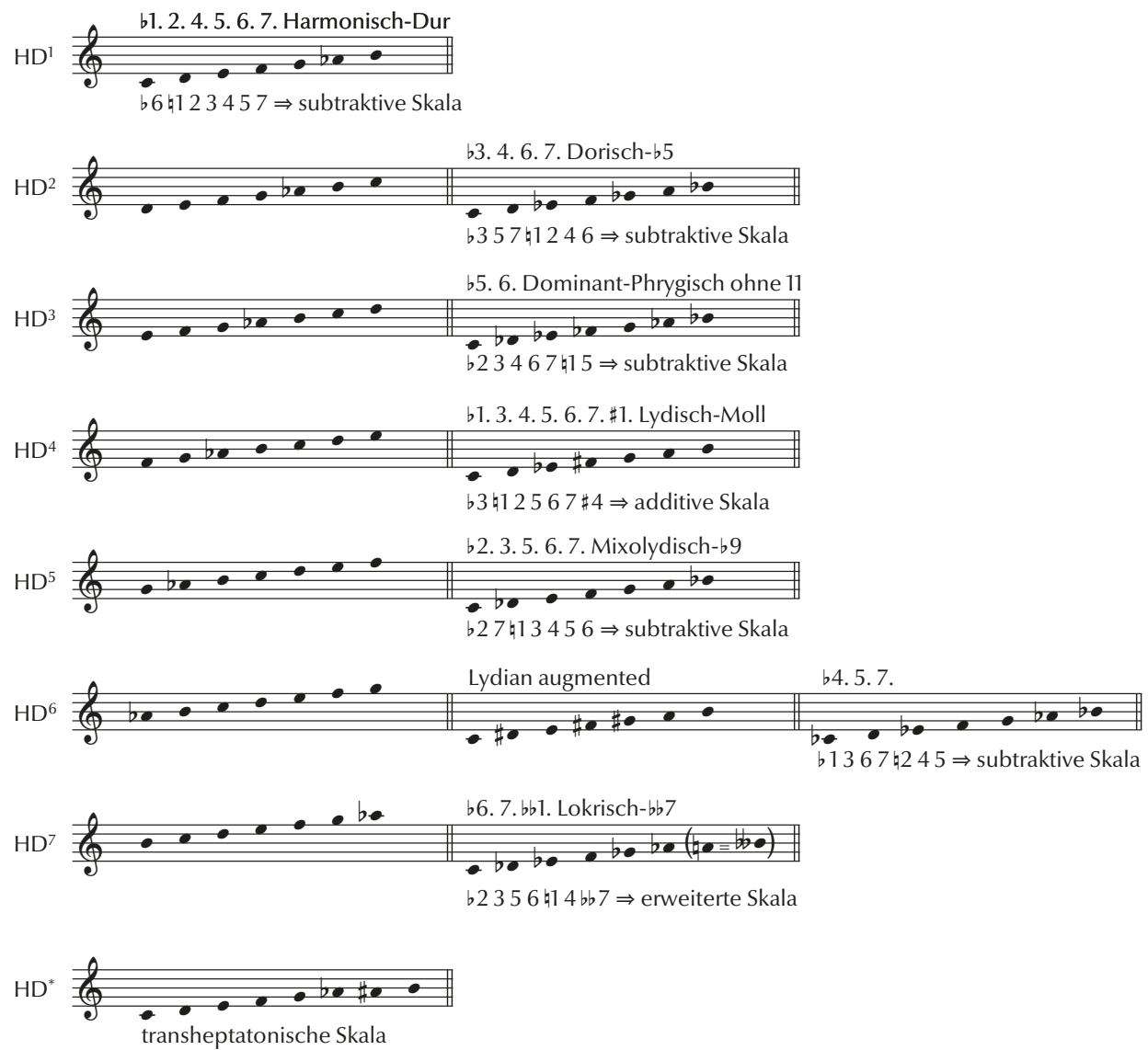

Beispiel 9: `Harmonisch-Dur 

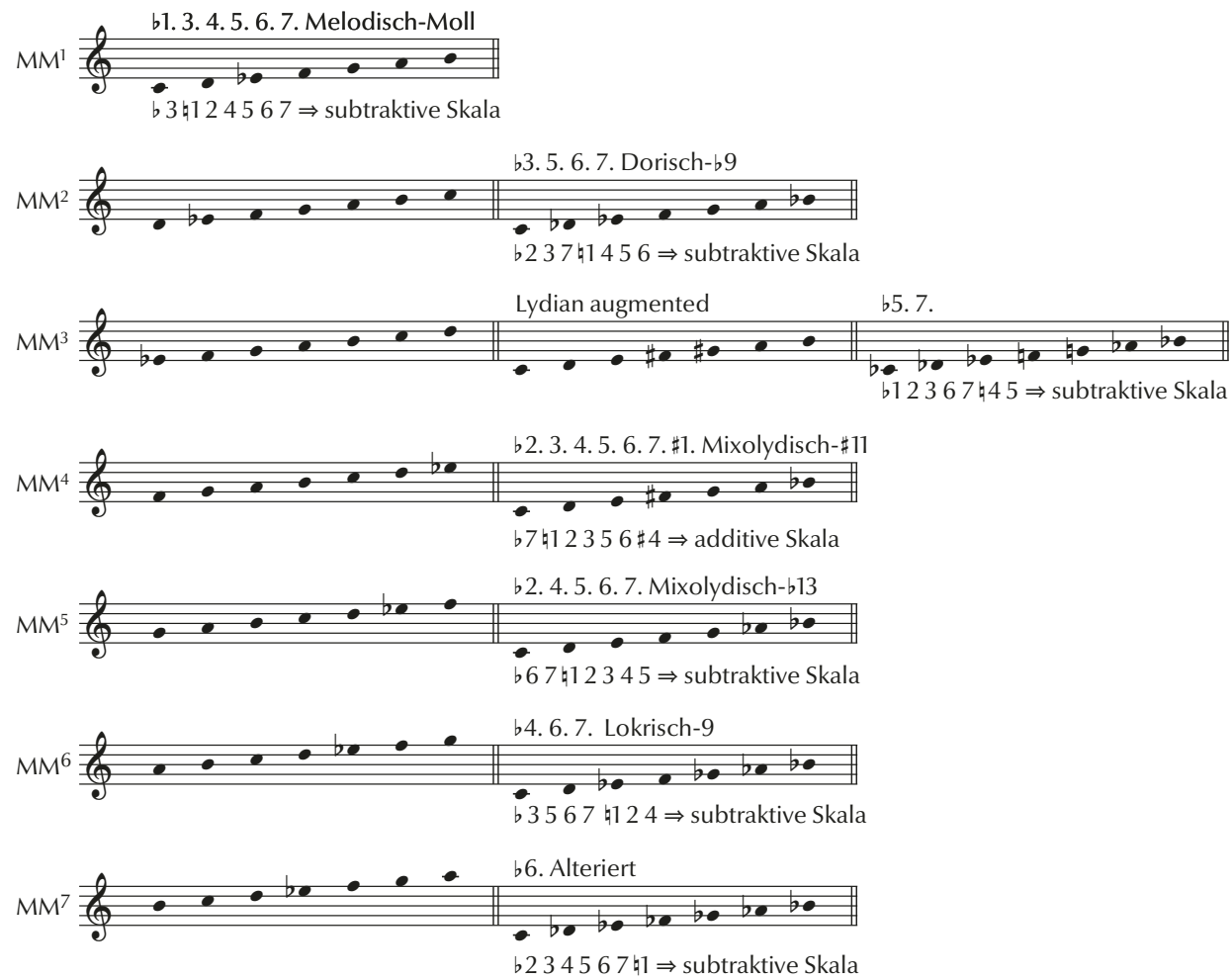

Beispiel 10: 'Melodisch-Molk

\section{Gilchrist modes}

Das benannte Auseinanderbrechen von traditionellen Skalengruppen innerhalb der vorgelegten Systematik wird auch an den »modes« deutlich, die Annie Gilchrist 1911 zur Erklärung gälischer Folksongs vorstellte (Bsp. 11).$^{90}$ Gilchrists Darstellung zeigt hexatonische Skalen, die aus der Pentatonik durch Hinzufügung von Tönen entstanden sind. Sie ging von den fünf halbtonlosen pentatonischen Skalen aus (Gilchrist modes 1-5; Gilchrist zählte sie anders als in der oben angeführten Tradition ${ }^{91}$ ) und füllte je eine der zwei kleinen Terzen durch einen nicht-alterierten Ton der diatonischen Stammtonreihe auf. So entstanden aus jeder der pentatonischen Skalen zwei verschiedene hexatonische Skalen, die Gilchrist dann als A- und B-Varianten bezeichnete. Im hier verfolgten Sprach-

90 Vgl. Gilchrist 1911, gut dargestellt bei Powers/Wiering/Porter/Cowdery/Widdess/Davis/Perlman/ Jones/Marett 2001, 825f.

91 Vgl. Bsp. 11 und oben, Abschnitt Anhemitonische Pentatonik. 

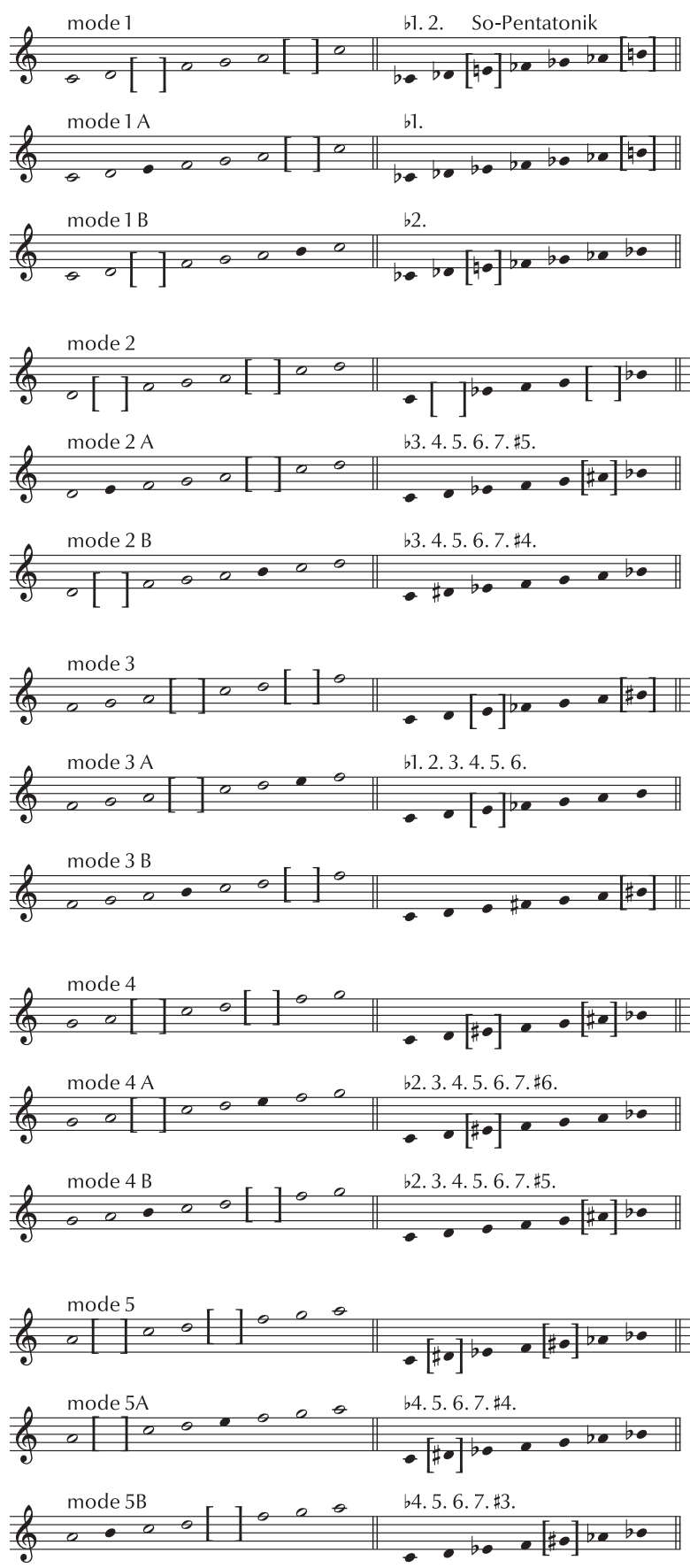

Beispiel 11: Gilchrist modes 
gebrauch sind dies zwar alles reduzierte Skalen, aber von verschiedener Art. Diese Arten lassen sich wie folgt differenzieren: Gilchrists mode 1 (ces-des-[e]-fes-ges-as-[h]) ist eine subtraktive Skala mit zwei eliminierten Tönen durch die Alteration b1.2., sie entspricht der `So-Pentatonikı. Gilchrists mode 1A (ces-des-es-fes-ges-as-[h]) korrespondiert derjenigen subtraktiven Skala mit einem eliminierten Ton, die durch die Alteration b1. entstanden ist; folglich handelt es sich um eine hexatonische reduzierte Skala subtraktiven Ursprungs. Gilchrists mode $1 B$ (ces-des-[e]-fes-ges-as- $b$ ), entstanden durch b2., ist ebenfalls um einen Ton reduziert. Mode $2 A(c-d$-es-f-g-[ais]-b) hingegen lässt sich nur als additive Skala mit einem eliminierten Ton erklären; es fehlt die 6. Stufe, der Ton ais ist durch Vereinigung mit $b$ klanglich eliminiert; es handelt sich folglich auch hier um eine hexatonische reduzierte Skala, aber additiven Ursprungs. ${ }^{92}$ Der Gilchrist mode $2 B$ gehört ebenfalls in diese Kategorie. Mode $3 B$ ist erneut eine hexatonische reduzierte Skala subtraktiven Ursprungs: b1.7. Die Gilchrist modes $4 A$ und $4 B$ wiederum sind hexatonische reduzierte Skalen additiven Ursprungs. Modes $5 A$ und $5 B$ sind additiven Ursprungs. Das Auseinanderbrechen dieser Skalengruppe innerhalb der hier vorgestellten Systematik geschieht, weil Gilchrist ein Töne hinzufügendes, auf Stufen- und nicht auf Alterationsebene operierendes Verfahren, ausgehend von der Pentatonik, gewählt hat - ein Verfahren, das der hier gewählten Denkrichtung entgegengesetzt ist.

\section{EINE SYSTEMATIK DIATONISCHER SKALEN: ZUSAMMENFASSUNG UND AUSBLICK}

Die hier vorgestellte Systematik bietet eine Klassifikation von Skalen, bei der zunächst durch ein subtraktiv-additives Alterationsverfahren einfache diatonische Skalen entstehen. Als einfache diatonische Skalen werden subtraktive und additive Skalen bezeichnet, die Skalen ersten und zweiten Grades. Durch verschiedene Arten des Wegfalls von Tönen (Elimination, Tilgung oder Streichung) entstehen Skalen, denen einzelne Töne fehlen, die also weniger als sieben Töne umfassen. Sie bilden eine dritte Klasse, die reduzierten Skalen oder Skalen dritten Grades. Wenn nun diesen reduzierten Skalen andere als die weggefallenen Töne hinzugefügt werden, entstehen erweiterte Skalen, die Skalen vierten Grades. Erweiterte Skalen mit weniger als sieben Tönen werden ebenfalls den reduzierten Skalen zugerechnet. Als Oberbegriff für reduzierte und erweiterte Skalen bietet sich im Gegensatz zu seinfache Skalen der Begriff skomplexe Skalen an. ${ }^{93}$ Die erweiterten Skalen charakterisieren das Übergangsfeld hin zu den transheptatonischen Skalen, die durch einen Vorrat von mehr als sieben Tönen gekennzeichnet sind.

Diatonische Skalen können innerhalb des Systems von der Ces-Dur-Skala ausgehend durch vier Operationen gebildet werden: erstens durch Erhöhung einzelner Skalentöne

92 Die Darstellung von ces aus würde ein Doppel-b erfordern (ces-des-eses) und wäre deshalb gemäß den aufgestellten Regeln weniger seinfach als die additive Darstellung.

$93 \mathrm{Vgl}$. in der Neurologie die Klassifikation der Epilepsien mithilfe der Begriffe ‘einfach - von daher entlehne ich diese Begriffe. Das Substantiv `Komplex` mit der Bedeutung `Verbund ‘ von Häusern, Objekten, Vorstellungen, Erlebnisweisen, Strukturen z.B. in der Architektur oder in der analytischen Psychologie ist hier nicht gemeint. 
um eine übermäßige Prime (Skalen ersten Grades, subtraktive Skalen), zweitens durch Aufwärtsalteration einzelner Töne um eine doppelt übermäßige Prime (Skalen zweiten Grades, additive Skalen), drittens durch Wegfall von Tönen (Skalen dritten Grades, reduzierte Skalen) und viertens durch Hinzufügung von Tönen, nachdem andere weggefallen sind (Skalen vierten Grades, erweiterte Skalen; wenn mehr Töne weggefallen als hinzugefügt sind, sind es reduzierte Skalen erweiterten Ursprungs).

Zur systematischen Benennung müssen Skalen, wenn möglich, auf ces transponiert werden, andernfalls auf $c$ oder cis. Bezeichnet werden die Skalenstufen mit den Stammtönen $c-d-e-f-g-a-h$ und den Akzidenzien $b$ und \# (oder ggf. mit den Doppelvorzeichen bb und \#\#). Mithilfe der Tonanzahl und der Zusatzzeichen für Alteration, Wegfall und Erweiterung wird sichtbar, in welche Skalenklasse eine Skala gehört: Subtraktive Skalen sind heptatonisch und durch $b$ - und heptatonisch und enthalten zudem \#-Vorzeichen, reduzierte Skalen enthalten weniger als sieben Töne, was durch eckige Klammern [ ] kenntlich gemacht wird (aber auch alle anderen Zeichen kommen in dieser Klasse vor), erweiterte Skalen sind wieder heptatonisch und man findet neben den bisher genannten Zeichen auch +-Zeichen und/oder Doppelvorzeichnung. Der Alterationsprozess wird durch Angabe derjenigen Vorzeichen markiert, die weggestrichen oder hinzugefügt wurden (jeweils mit Punkt), die resultierende Skala durch die Angabe der Alterationsart der jeweiligen Stufen (z.B. im Fall von Phrygisch c-des-es-f-g-as- $b$ : Alterationsprozess ausgehend von Ces-Dur b5.6.7.; Ergebnis 1 h, $2 b, 3 b, 4$ h, 5 h, $6 b, 7 b$ oder b2367 1445 ).

Das Verfahren geht von der Pedalharfe und ihren Alterationsmöglichkeiten bzw. den geläufigen gleichstufig und heptatonisch orientierten Notations- und Denkkonventionen aus. Es bietet eine Möglichkeit, alle halbtönig-gleichstufigen Skalen bis zur Heptatonik (und etwas darüber hinaus) zu klassifizieren: als einfache, also subtraktive oder additive Skalen sowie als komplexe, also reduzierte und erweiterte Skalen. Festzuhalten ist, dass im Verlauf der obigen Diskussion dieser Skalensystematik Übergangsbereiche zwischen den Skalenklassen deutlich geworden sind, in denen die Grenzen zwischen Kategorien/ Klassen sich nicht aus dem System selbst ergeben, sondern nur argumentativ definiert und gebildet werden konnten. Die Dichotomie von Diatonik und Chromatik wurde dabei relativiert, indem sdiatonisch den. ${ }^{94}$ Am Begriff der >chromatischen Skalas als einer einzigen wurde festgehalten. Die Grenzen der entwickelten Systematik wurden aufgezeigt.

Die Klassifikation kann nun weiter differenziert werden, wenn man eingangs definierte Voraussetzungen aufhebt: Wird etwa die Tatsache der Oktavidentität nicht mehr als Begrenzung akzeptiert, ergeben sich Möglichkeiten, kombinierte Skalen zu bilden, die sich über den Oktavraum hinaus erstrecken, wobei die Skalenausschnitte in der ersten und zweiten Oktave voneinander verschieden sind. Wenn die gleichstufige Stimmung verlassen wird, können weitere Skalen systematisiert werden wie z.B. arabische maqam at, indische ragas, slendro und pelog in Gamelan-Musik oder empirisch dokumentierte Formen der erwähnten chinesischen Pentatonik. Die Grenze zwischen Stimmungssystemen und Skalen- bzw. Stufensystemen würde so fließend und unter Umständen zu einem Kontinuum:

94 Siehe oben, Anm. 42 und 43. 
So könnte man diskutieren, ob «Kirchentonarten Skalen darstellen als in der gleichstufigen oder nicht. Dieselbe Frage stellt sich z.B. bei arabischen Skalen - für die jüdische Tradition hat Abraham Z. Idelsohn bereits eine aus der Praxis kommende Antwort geliefert. ${ }^{95}$ Es folgt daraus, dass alle bisher besprochenen Skalen ıgleichstufige ‘ genannt werden müssen und andere ınicht-gleichstufige ‘.

Unter Berücksichtigung derlei möglicher Erweiterungen kommt man schließlich zu folgender Klassifikationsystematik.

\begin{tabular}{|c|c|c|c|c|}
\hline \multirow[t]{14}{*}{ Gleichstufige Skalen } & $\begin{array}{l}\text { Gleichstufige } \\
\text { Skalen auf Viertel-, } \\
\text { Sechstel-, Achtel- } \\
\text { tonbasis }\end{array}$ & & & \\
\hline & \multirow{12}{*}{$\begin{array}{l}\text { Gleichstufige } \\
\text { Skalen auf Halb- } \\
\text { tonbasis }\end{array}$} & \multirow{3}{*}{$\begin{array}{l}\text { Einfache Skalen } \\
\text { (diatonischer } \\
\text { Herkunft) }\end{array}$} & \multirow[t]{2}{*}{$\begin{array}{l}\text { Heptatonische subtraktive } \\
\text { Skalen (Skalen ersten Grades) }\end{array}$} & $\begin{array}{l}\text { Skalen mit kontinuierlicher } \\
\text { Alteration }\end{array}$ \\
\hline & & & & $\begin{array}{l}\text { Skalen mit diskontinuierlicher } \\
\text { Alteration }\end{array}$ \\
\hline & & & $\begin{array}{l}\text { Heptatonische additive Skalen } \\
\text { (Skalen zweiten Grades) }\end{array}$ & \\
\hline & & \multirow{7}{*}{$\begin{array}{l}\text { Komplexe Skalen } \\
\text { (diatonischer } \\
\text { Herkunft) }\end{array}$} & \multirow{4}{*}{$\begin{array}{l}\text { Reduzierte Skalen (Skalen } \\
\text { dritten Grades) }\end{array}$} & Tritonische Sklaen \\
\hline & & & & Tetratonische Skalen \\
\hline & & & & $\begin{array}{l}\text { Pentatonische Sklaen } \\
\text { a) subtraktiven Ursprungs } \\
\text { b) additiven Ursprungs } \\
\text { c) erweiterten Ursprungs } \\
\text { - mit getilgten Tönen } \\
\text { (Doppelvorzeichnung) } \\
\text { - mit gestrichenen und } \\
\text { hinzugefügten Tönen }\end{array}$ \\
\hline & & & & $\begin{array}{l}\text { Hexatonische Skalen (einfach } \\
\text { reduzierte Skalen) } \\
\text { a) subtraktiven Ursprungs } \\
\text { b) additiven Ursprungs } \\
\text { c) erweiterten Ursprungs } \\
\text { - mit getilgten Tönen } \\
\text { (Doppelvorzeichnung) } \\
\text { - mit gestrichenen und } \\
\text { hinzugefügten Tönen }\end{array}$ \\
\hline & & & \multirow{2}{*}{$\begin{array}{l}\text { Heptatonische erweiterte } \\
\text { Skalen (Skalen vierten } \\
\text { Grades) }\end{array}$} & $\begin{array}{l}\text { Skalen mit hinzugefügten } \\
\text { Tönen }\end{array}$ \\
\hline & & & & Skalen mit ergänzten Tönen \\
\hline & & & Transheptatonische Skalen & \\
\hline & & Chromatische Skala & & \\
\hline & & Kombinierte Skalen & & \\
\hline & $\begin{array}{l}\text { Gleichstufige } \\
\text { Skalen auf Drei- } \\
\text { vierteltonbasis }\end{array}$ & & & \\
\hline $\begin{array}{l}\text { Nicht-gleichstufige } \\
\text { Skalen }\end{array}$ & & & & \\
\hline
\end{tabular}

Tabelle 1: Skalensystematik (Überblick) ${ }^{96}$

95 Siehe oben, Anm. 64.

96 Zu den gleichstufigen Skalen auf Dreivierteltonbasis vgl. Lemke 2017, 55. 
Die vorgestellte Art der Systematisierung erhebt nicht den Anspruch, in der musikalischen Praxis etablierte und bewährte Wege der Bildung von und des Umgangs mit Skalen zu verdrängen. Doch bietet sie eine systematisch konsistente Methode, (auch bisher unbenannte) Skalen zu klassifizieren und den Blick dafür zu schärfen, wie Skalen in der kompositorischen und analytischen Praxis verwendet werden können. Weiterer Forschung und Komposition bliebe es etwa vorbehalten, Skalen und deren Obertonstruktuals spektrale Farben zu untersuchen und mit diesen Aspekten gezielt zu arbeiten ${ }^{97}$, ein Verfahren, das sich vielleicht als "new modality« bezeichnen ließe, in Orientierung an Praktiken der elektroakustischen und akusmatischen Musik, wo dieser Begriff im Zusammenhang von »sound objects as modes« und »sonic magnification ${ }^{98}$ bereits verwendet worden ist.

97 Meine Orgelkomposition Meditation versucht dies unter Verwendung der subtraktiven Skalen zu erproben.

98 Richards 2001, [1]. 


\section{ANHANG: GESAMTVERZEICHNIS SUBTRAKTIVER SKALEN}

128 subtraktive Skalen

Cb-Dur/lonisch

b2. Gilchrist Mode 1B b3.

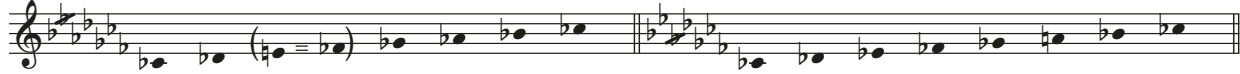

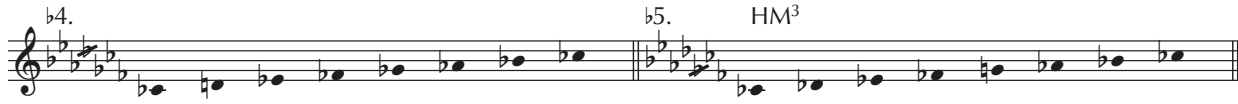

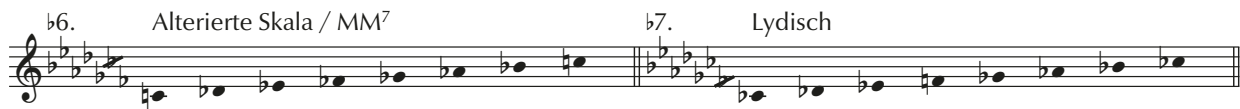

b1. 2. So-Pentatonik / Gilchrist Mode 1

b1. 3.

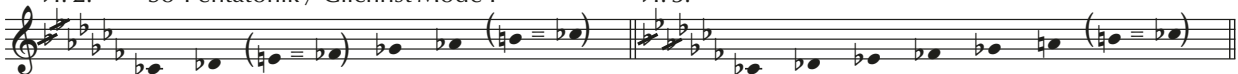

b1. 4.

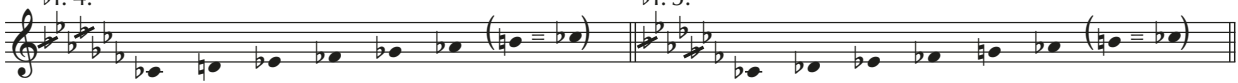

b1. 7.

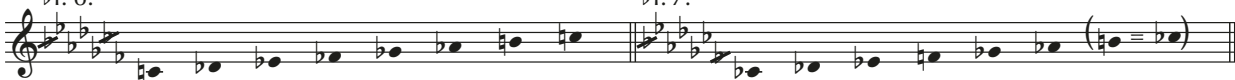

2. 3.

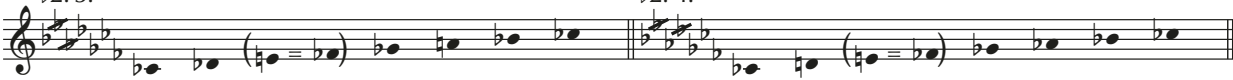

b2. 5 . b2. 6.

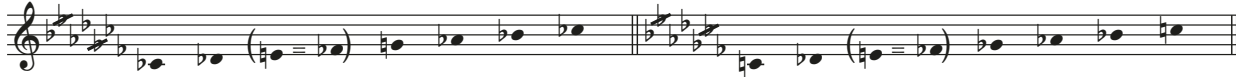

b2.7. b3. 4

(a)

b3. 5.

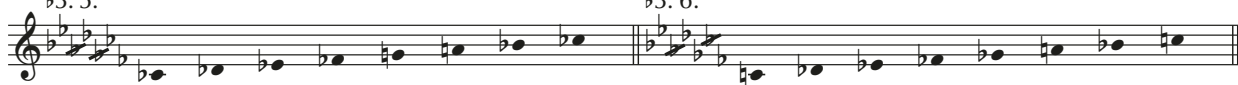

b3. 7.

b4. 5 .

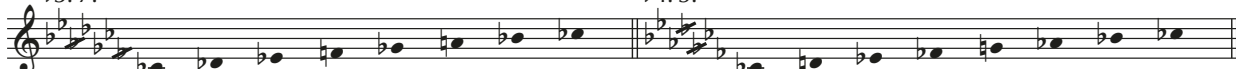

Beispiel 12: Subtraktive Skalen (vollständige Auflistung) 


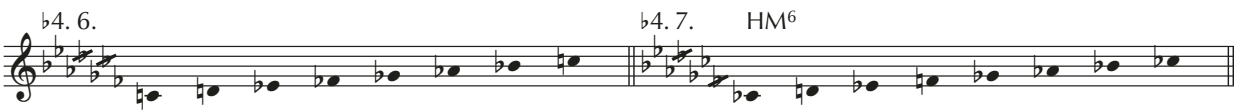
(be ${ }^{b 5 .} \mathrm{HD}^{3}$

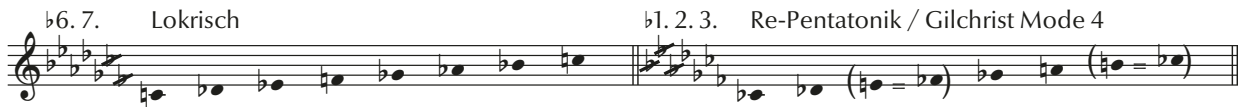
b1. 2. $4 . \quad$ b1. 2.5. Hemitonische Pentatonik

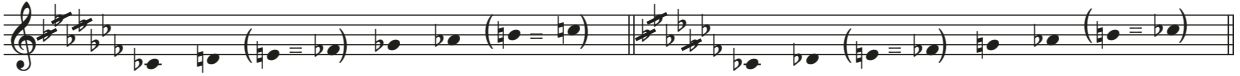
b1. 2. $6 . \quad$ b1. 2.7 .

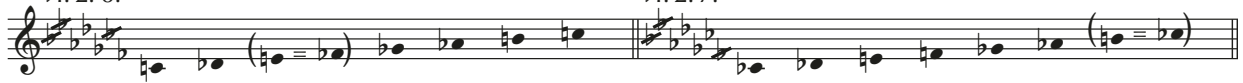
b1. 3.4 .

b1. 3. 5 .

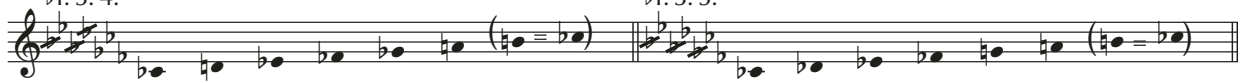
b1. 3.6 .

b1. 3. 7 . $\int^{b} \frac{b}{b} b_{0} b_{0} b_{0} b_{0} b_{0} b_{0}$ b1. 4.5 .

b1. 4.6 .

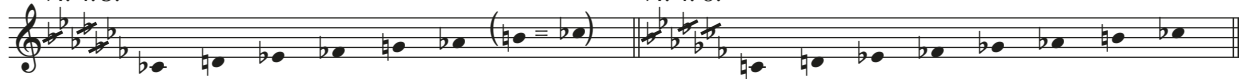
b1. 4.7 .

b1. 5.6 .

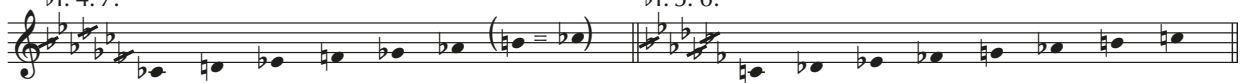
b1. 5.7 .

b1. 6.7 .

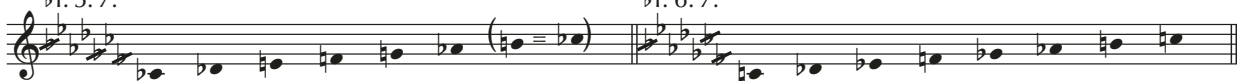
b2. 3. 4.

b2.3. 5 .

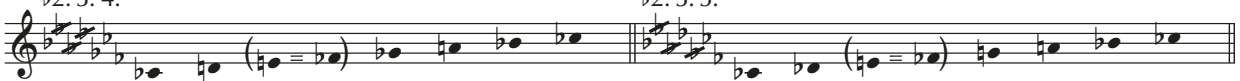

b2.3. 6 .

b2.3. 7 .

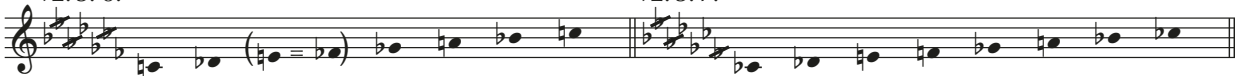

b2. 4. 5 .

b2. 4. 6. Ganztonskala

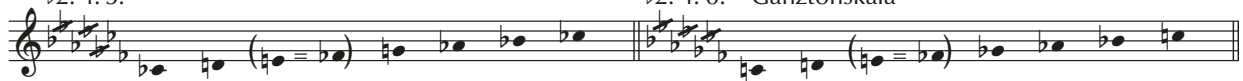

Beispiel 12 (Fortsetzung) 
b2. 4.7 .

b2.5.6.

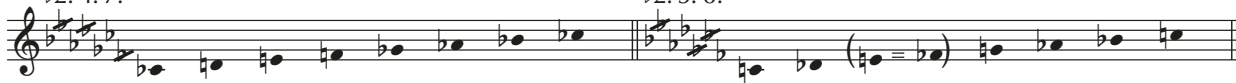

b2.5.7.

b2. 6.7 .

$\int_{0}^{b} b_{b}^{b} \frac{b}{b 0}$

b3.4.5. b3. 4. 6 .

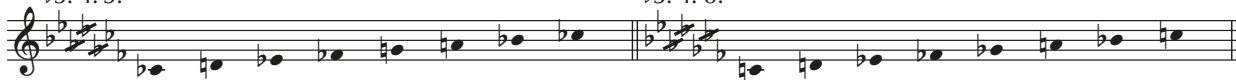

b3. 4.7. b3. 5.6 .

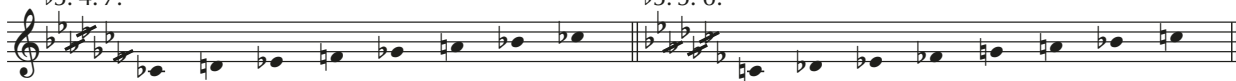

$\begin{array}{ll}\text { b3. } 5.7 . & \text { b3.6.7. } \mathrm{HM}^{2}\end{array}$

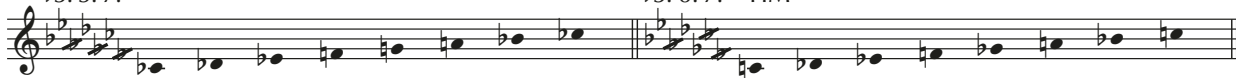

b4. 5. 6. b4. 5.7. $\mathrm{HD}^{6}$

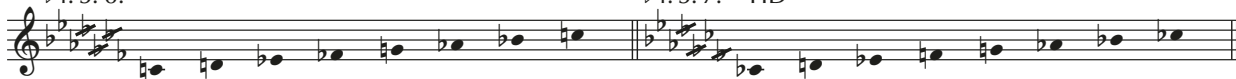

b4.6.7. $\mathrm{MM}^{6}$ b5.6.7. Phrygisch

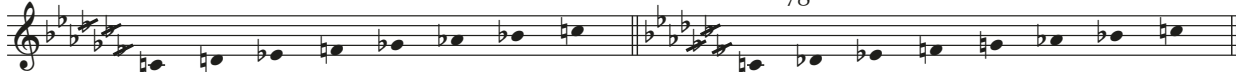

b1. 2. 3. 4. La-Pentatonik / Gilchrist Mode 2

b1. 2. 3. 5. Hemitonische Pentatonik

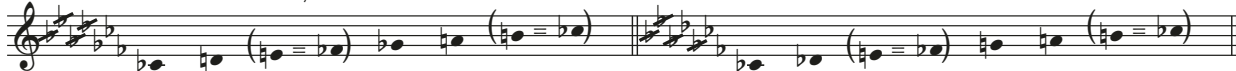

b1. 2.3. 6 .

b1. 2.3. 7 .

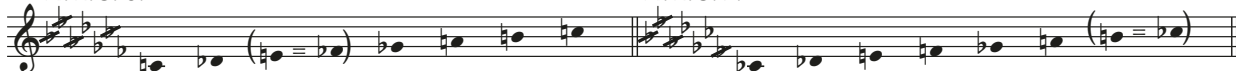

b1. 2. 4. 5. Hemitonische Pentatonik

b1. 2. 4. 6 .

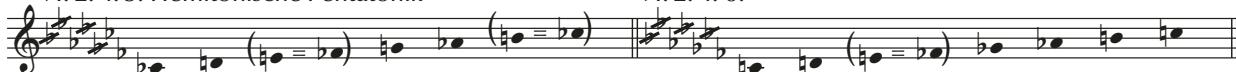

b1. 2. 4. 7. Gilchrist Mode 5A

b1. 2. 5. 6 .

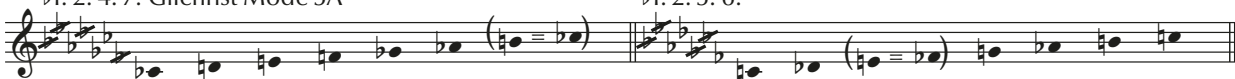

b1. 2.5.7.

b1. 2. 6.7 .

$\left(\frac{0}{b} b_{b}^{b} b_{0} b_{0}\right.$

b1. 3. 4.5 .

b1. 3. 4. 6 .

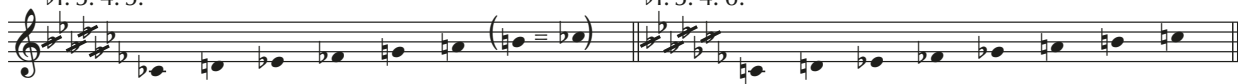

Beispiel 12 (Fortsetzung) 
b1. 3. 4. 7 .

b1.3.5. 6 .

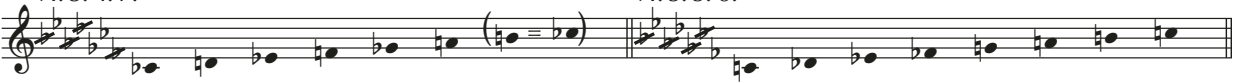

b1. 3. 5.7. Ganztonskala

b1. 3. 6. 7 .

$\int^{b} \frac{b}{b} b_{0}^{b} b_{0}$

$\begin{array}{ll}\text { b1. 4. } 5.6 . & \text { b1. 4. } 5.7 .\end{array}$

(a)

b1. 5. 6.7. b1. 4. 6.7 .

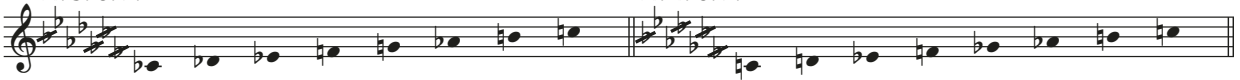

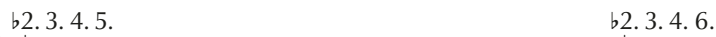

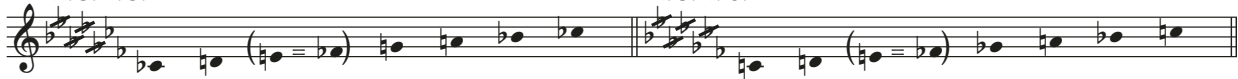

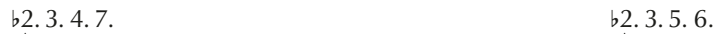

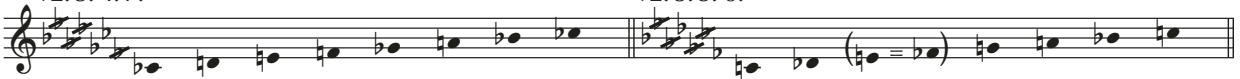

b2.3.5.7. b2.3.6.7.

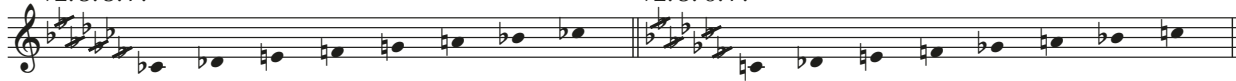

b2. 4.5.6. b2. 4.5 .7 .

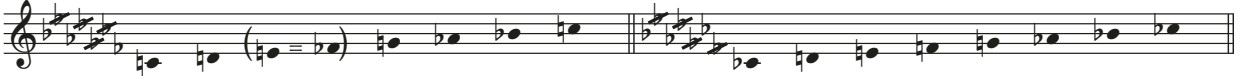

b2. 4.6.7. b2.5.6.7. Phrygisch-dominante Skala / $\mathrm{HM}^{5}$

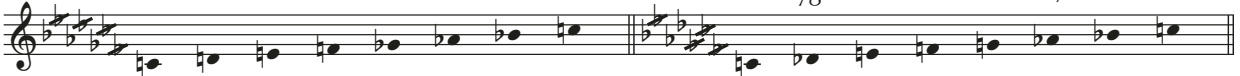
b3. 4. 5. 6 .
b3. 4. 5.7.

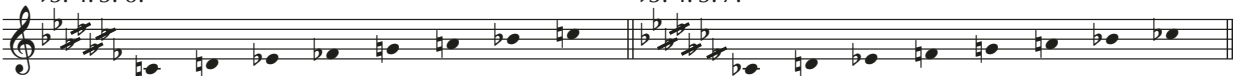

b3. 4.6.7. $\mathrm{HD}^{2}$

b3. 5. 6. 7. $\mathrm{MM}^{2}$

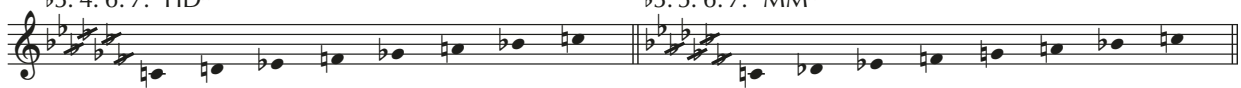

b4. 5. 6. 7. Äolisch / Moll / melodisch Moll abwärts b1. 2. 3. 4. 5. Mi-Pentatonik / Gilchrist Mode 5

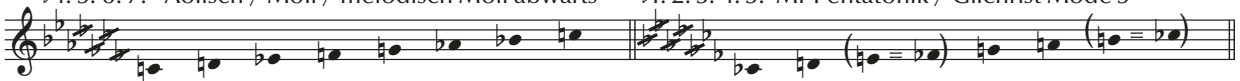

b1. 2. 3. 4. 6 .

b1. 2. 3. 4.7 .

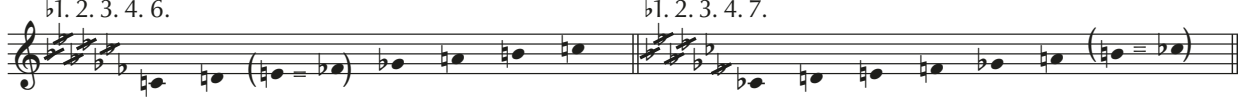

Beispiel 12 (Fortsetzung) 
b1. 2.3.5. 6

b1. 2. 3. 5. 7 .

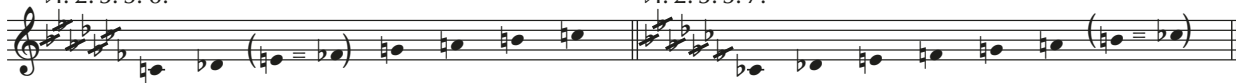

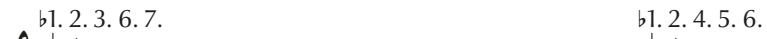

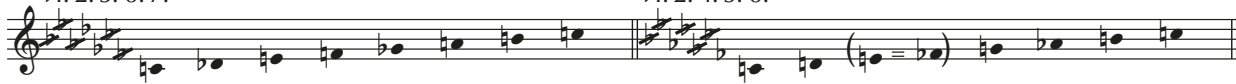

b1. 2. 4. 5.7. b1. 2. 4.6.7.

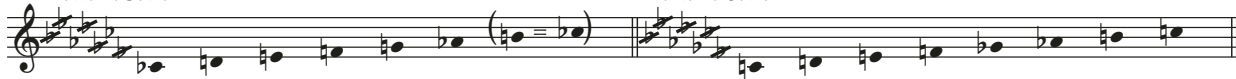

b1. 2.5.6.7. 'Zigeuner-Dur / Fraigisch b1.3.4.5.6.

(a)

b1.3.4.5.7. b1.3. 4. 6.7 .

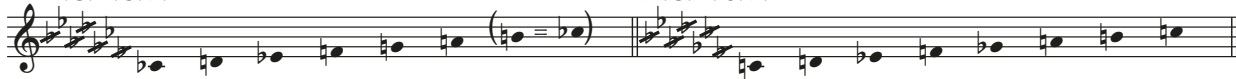

b1. 3. 5.6.7.

b1. 4. 5. 6. 7. Harmonisch Moll HMำ

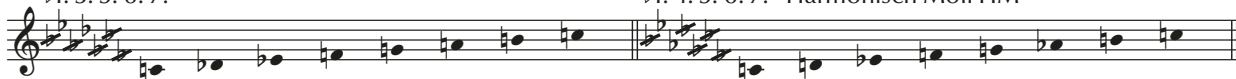

b2. 3. 4. 5. 6 .

b2. 3. 4.5.7.

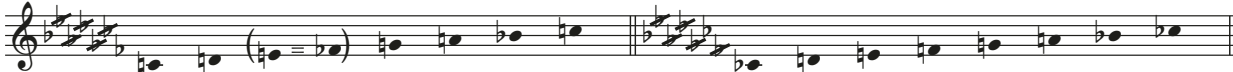

b2. 3. 4.6.7.

b2.3.5.6.7. $\mathrm{HD}^{5}$

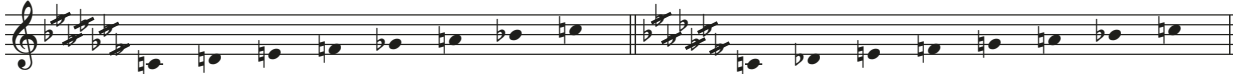

b2. 4. 5.6.7. $\mathrm{MM}^{5}$

b3. 4. 5. 6.7. Dorisch

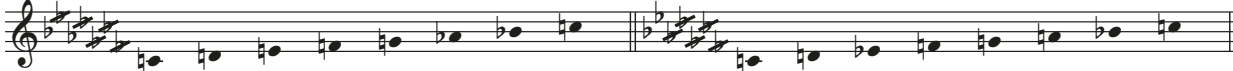

b1. 2. 3. 4. 5. 6. Gilchrist Mode 3A

b1. 2.3.4.5.7.

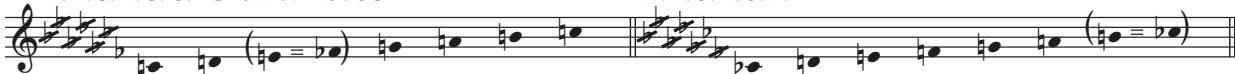

b1. 2. 3. 4.6.7.

b1. 2. 3. 5.6.7.

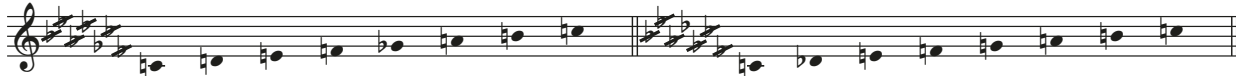

b1. 2. 4. 5. 6. 7. Harmonisch-Dur $\mathrm{HD}^{1}$

b1. 3. 4.5.6.7.

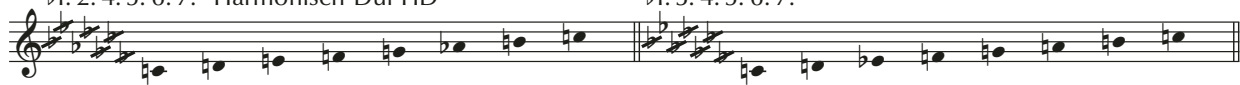

b2. 3. 4. 5. 6.7. Mixolydisch

b1. 2. 3. 4. 5. 6. 7. C-Dur / Ionisch $\triangleq b 0 .(C b$-Dur $)$

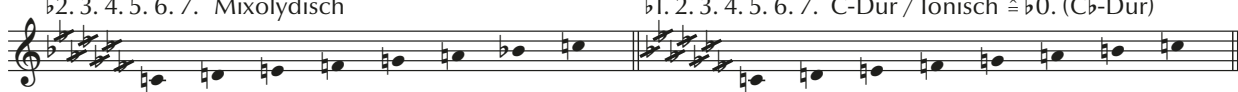

Beispiel 12 (Fortsetzung) 


\section{Literatur}

Amon, Reinhard (2005), Lexikon der Harmonielehre. Nachschlagewerk zur durmolltonalen Harmonik mit Analysechiffren für Funktionen, Stufen und Jazz-Akkorde, Wien: Doblinger.

Annewanter, Gerhard (1977), »Isländische Zwiegesänge (Tvisöngur, Tvisöngvar)«, in: Jahrbuch für musikalische Volks- und Völkerkunde, Bd. 8, hg. von Josef Kuckertz, Köln: Gerig, 12-35.

Antokoletz, Elliott (1984), The Music of Béla Bartók: A Study of Tonality and Progression in Twentieth-Century Music, Berkeley (CA): University of California Press.

Bartók, Béla (1976), »Harvard Lectures« [1943], in: ders., Essays, hg. von Benjamin Suchoff, London: Faber \& Faber, 354-392.

Bauer, Susan (1999), Von der Khupe zum Klezkamp. Klezmer-Musik in New York, Berlin: Piranha.

--- (2002), von der Khupe zum KlezKamp. http://www.klezmer.de/Buecher/S_KhupeInhalt/s_khupe-inhalt.html (31.12.2017)

Brieger, Jochen (2010a), »Modus«, in: Lexikon der Systematischen Musikwissenschaft (= Handbuch der Systematischen Musikwissenschaft, Bd. 6), hg. von Helga de la Motte-Haber, Heinz von Loesch, Günther Rötter und Christian Utz, Laaber: Laaber, 297-301.

(2010b), Untersuchungen zur Struktur der Erstsoggetti in den Motetten Giovanni Pierluigi da Palestrinas, Göttingen: Vandenhoek \& Ruprecht.

Bühler, Walter (2014), Rechnen mit musikalischen Intervallen, Skalen und Stimmungen im historischen Kontext, Frankfurt a.M.: Lang.

Cahn, Peter (1995), „Diatonik - Chromatik - Enharmonik«, in: Die Musik in Geschichte und Gegenwart. Allgemeine Enzyklopädie der Musik, 2. Auflage, hg. von Ludwig Finscher, Sachteil Bd. 2, Kassel: Bärenreiter / Stuttgart: Metzler, 1213-1234.

Calella, Michele (2005), »Parallele (Welt-)Räume. Bemerkungen zur Entwicklung des Tonsystems zwischen Mittelalter und Neuzeit", in: Musik und Raum. Dimensionen im Gespräch, hg. von Annette Landau und Claudia Emmenegger, Zürich: Chronos, 81-91.

Chen, Yingshi (2002), »Theory and Notation in China«, in: East Asia: China, Japan, and Korea (= The Garland Encyclopedia of World Music, Bd. 7), hg. von Robert C. Provine, Yosihiko Tokumaru und J. Lawrence Witzleben, New York: Routledge, 115-126.

Cowell, Henry (1996), New Musical Resources [1930], hg. von David Nicholls, Cambridge: Cambridge University Press.

Dahlhaus, Carl (1998), »Tonalität«, in: Die Musik in Geschichte und Gegenwart. Allgemeine Enzyklopädie der Musik, 2. Auflage, hg. von Ludwig Finscher, Sachteil Bd. 9, Kassel: Bärenreiter / Stuttgart: Metzler, 623-628.

(1966), »Systematik«, »Europäisch« (= »Tonsysteme« A und B), in: Die Musik in Geschichte und Gegenwart. Allgemeine Enzyklopädie der Musik, 1. Auflage, hg. von Friedrich Blume, Bd. 13, Kassel: Bärenreiter, 533-547. 
Dahlhaus, Carl / Hans-Peter Reinecke / Marius Schneider (1966), „Tonalität«, in: Die Musik in Geschichte und Gegenwart. Allgemeine Enzyklopädie der Musik, 1. Auflage, hg. von Friedrich Blume, Bd. 13, Kassel: Bärenreiter, 510-522.

Drabkin, William (2001), "Scale», in: The New Grove Dictionary of Music and Musicians, 2. Auflage, hg. von Stanley Sadie, Bd. 22, London: Macmillan, 366-367.

Eimert, Herbert / Hans Ulrich Humpert (1973), Lexikon der elektronischen Musik, Regensburg: Bosse.

Eybl, Martin (2010), „Tonalität«, in: Lexikon der Systematischen Musikwissenschaft (= Handbuch der Systematischen Musikwissenschaft, Bd. 6), hg. von Helga de la Motte-Haber, Heinz von Loesch, Günther Rötter und Christian Utz, Laaber: Laaber, 485-488.

Gemoll, Wilhelm (1937), Griechisch-deutsches Schul- und Handwörterbuch [1908], 4. Auflage, Berlin: Freytag.

Gilchrist, Annie G. (1911), »Note on the Modal System of Gaelic Tunes«, Journal of the Folk-Song Society 4/16, 150-153.

Gilles, Malcolm (1982), »Bartók's Last Works. A Theory of Tonality and Modality«, Musicology Australia 7/1, 120-130.

Gissel, Siegfried (2013), Die Tonarten in mehrstimmigen Messen von Ockeghem bis Palestrina, Bd. 1, Wilhelmshaven: Noetzel.

Gunther, Leon (2012), The Physics of Music and Color, New York: Springer.

Haunschild, Frank (1992), Die neue Harmonielehre. Ein musikalisches Arbeitsbuch für Klassik, Rock, Pop und Jazz, Bd. 2, Brühl: AMA.

- (1997), Die harmonischen Grundlagen (= Die neue Harmonielehre. Ein musikalisches Arbeitsbuch für Klassik, Rock, Pop und Jazz, Bd. 1) [1988], erweiterte und überarbeitete Neuauflage, Brühl: AMA.

Heinemann, Michael (2010), „Tonsatz als Metapher«, in: Musik und ihre Theorien. Clemens Kühn zum 65. Geburtstag, hg. von Felix Diergarten, Ludwig Holtmeier, John Leigh und Edith Metzner, Dresden: Sandstein, 221-224.

Heygster, Malte / Manfred Grunenberg (1998), Handbuch der relativen Solmisation, Mainz: Schott.

Holtmeier, Ludwig (2010a), „Skala«, in: Lexikon der Systematischen Musikwissenschaft (= Handbuch der Systematischen Musikwissenschaft, Bd. 6), hg. von Helga de la Motte-Haber, Heinz von Loesch, Günther Rötter und Christian Utz, Laaber: Laaber, 430-432.

- (2010b), „Vom Triebleben der Stufen. Gedanken zum Tonalitätsbegriff Arnold Schönbergs", in: Musik und ihre Theorien. Clemens Kühn zum 65. Geburtstag, hg. von Felix Diergarten, Ludwig Holtmeier, John Leigh und Edith Metzner, Dresden: Sandstein, 84-108.

Hüschen, Heinrich (1961), »Modus", in: Die Musik in Geschichte und Gegenwart. Allgemeine Enzyklopädie der Musik, 1. Auflage, hg. von Friedrich Blume, Bd. 9, Kassel: Bärenreiter / Stuttgart: Metzler, 402-414. 
Hyer, Brian (2001a), »Key« (i), in: The New Grove Dictionary of Music and Musicians, 2. Auflage, hg. von Stanley Sadie, Bd. 13, London: Macmillan, 509.

- (2001b), »Tonality«, in: The New Grove Dictionary of Music and Musicians, 2. Auflage, hg. von Stanley Sadie, Bd. 25, London: Macmillan, 583-594.

Idelsohn, Abraham Z. (1992), Jewish Music. Its Historical Development [1929], hg. von Arbie Orenstein, New York: Dover.

Jans, Markus (2013), »Modus und Modalität wahrnehmen und vermitteln. Über die Arbeit mit Tonfeldern. Ein Erfahrungsbericht«, in: Das modale System im Spannungsfeld zwischen Theorie und kompositorischer Praxis, hg. von Jochen Brieger, Frankfurt a.M.: Lang, 41-54.

Kahan, Sylvia (2009), In Search of New Scales: Prince Edmond de Polignac, Octatonic Explorer, Rochester (NY): University of Rochester Press.

Kissenbeck, Andreas (2007), Harmonik und Skalen (= Jazztheorie, Bd. 1), Kassel: Bärenreiter.

Komoda, Haruko / Mihoko Nogawa (2002), „Theory and Notation in Japan«, in: East Asia: China, Japan, and Korea (= The Garland Encyclopedia of World Music, Bd. 7), hg. von Robert C. Provine, Yosihiko Tokumaru und J. Lawrence Witzleben, New York: Routledge, 565-584.

Krüger, Eric (2011), Skalen der Musik. Entstehung, Analyse und Vergleich wichtigster Tonleitern. Praktische Gegenüberstellung, München: Meidenbauer.

Kruse, Johannes (2017), „Was ist Struktur? Psychodynamische Perspektive», Psychotherapeut $62 / 2,106-112$.

Kuhn, Hans Niklas (2014), „Skalen in der klassischen Moderne. Béla Bartók: Klavierstücke», in: Musikalische Analyse. Begriffe, Geschichten, Methoden, hg. von Felix Diergarten, Laaber: Laaber, 237-265.

Lemke, Sascha Lino (2017), »»Hier ist alles Satz،. Zum `Finale` von Enno Poppes Wald für vier Streichquartette», in: Enno Poppe, hg. von Ulrich Tadday, München: edition text + kritik, 52-72.

Lendvai, Ernő (1957), »Einführung in die Formen- und Harmoniewelt Bartóks«, in: Béla Bartók. Weg und Werk. Schriften und Briefe, hg. v. Bence Szabolcsi, Kassel: Bärenreiter, 91-137.

Liang, Mingyue (1985), Music of the Billion. An Introduction to Chinese Musical Culture, New York: Heinrichshofen.

Lindley, Mark / Ronald F. Turner-Smith (1993), Mathematical Models of Musical Scales: A New Approach, Bonn: Verlag für systematische Musikwissenschaft.

Menke, Johannes (2015), Kontrapunkt I. Die Musik der Renaissance, Laaber: Laaber.

Messiaen, Olivier (1966), Technik meiner musikalischen Sprache (2 Bde.), Paris: Leduc. (2012), Traité de rythme, de couleur et d'ornithologie. Textauswahl in deutscher Übersetzung (= Texte, Analysen, Zeugnisse, Bd. 1), hg. von Wolfgang Rathert, Karl Anton Rickenbacher und Herbert Schneider, Hildesheim: Olms. 
Michaels, Axel (2005), »Rituelle Klangräume«, in: Musik und Raum. Dimensionen im Gespräch, hg. von Annette Landau und Claudia Emmenegger, Zürich: Chronos, 33-44.

Moßburger, Hubert (2012), Ästhetische Harmonielehre. Quellen · Analysen · Aufgaben (2 Bde.), Wilhelmshaven: Noetzel.

Petersen, Peter (1971), Die Tonalität im Instrumentalschaffen von Béla Bartók, Hamburg: Wagner.

Pöhlmann, Egert (1995), »Antike Musik» (= »Griechenland« A), in: Die Musik in Geschichte und Gegenwart. Allgemeine Enzyklopädie der Musik, 2. Auflage, hg. von Ludwig Finscher, Sachteil Bd. 3, Kassel: Bärenreiter / Stuttgart: Metzler, 1627-1676.

Powers, Cameron (2006), Arabic Musical Scales: Basic Maqam Notation, Boulder (CO): G. L. Design.

Powers, Harold S. / Frans Wiering / James Porter / James Cowdery / Richard Widdess / Ruth Davis / Marc Perlman / Stephen Jones / Allan Marett (2001), »Mode«, in: The New Grove Dictionary of Music and Musicians, 2. Auflage, hg. von Stanley Sadie, Bd. 16, London: Macmillan, 775-860.

Rambold, Alois (2012), Elementare Strukturen und Funktionen des musikalischen Satzes. Eine tonmengenbezogene, intervallorientierte Funktionstheorie und ihre Anwendung in der Analyse, Baden-Baden: Arion.

Rätz, Ricarda (2009), »Josef Matthias Hauers System der Tropen«, in: Musiktheorie an ihren Grenzen: Neue und Alte Musik. 3. Internationaler Kongress für Musiktheorie, 10.-12. Oktober 2003, Musik-Akademie der Stadt Basel, hg. von Angelika Moths, Markus Jans, John MacKeown und Balz Trümpy, Bern: Lang, 99-110.

Reinecke, Hans-Peter / Fritz Winckel (1966), „Ton«, in: Die Musik in Geschichte und Gegenwart. Allgemeine Enzyklopädie der Musik, 1. Auflage, hg. von Friedrich Blume, Bd. 13, Kassel: Bärenreiter, 488-509.

Rettelbach, Johannes (1998), »Ton«, in: Die Musik in Geschichte und Gegenwart. Allgemeine Enzyklopädie der Musik, 2. Auflage, hg. von Ludwig Finscher, Sachteil Bd. 9, Kassel: Bärenreiter / Stuttgart: Metzler, 618-620.

Richards, John (2001), New Modality: Sonic Magnification. http://www.dmu.ac.uk/ documents/technology-documents/research/mtirc/nowalls/mww-richards.pdf (31.12.2017).

Rings, Steven (2011), Tonality and Transformation, New York: Oxford University Press.

Rohringer, Stefan (2010), „Diatonik / Chromatik / Enharmonik«, in: Lexikon der Systematischen Musikwissenschaft (= Handbuch der Systematischen Naturwissenschaft, Bd. 6), hg. von Helga de la Motte-Haber, Heinz von Loesch, Günther Rötter und Christian Utz, Laaber: Laaber, 82-86.

Rossi, Mike (2007), Uncommon Etudes from Common Scales. Applying Melodic Contrast to Diatonic Phrases, Mainz: Schott.

Rufer, Josef (1966), Die Komposition mit zwölf Tönen [1952], 2. Auflage, Kassel: Bärenreiter.

Scharff, Jörg M. (2010), Die leibliche Dimension in der Psychoanalyse, Frankfurt a.M.: Brandes \& Apsel. 
Scheideler, Ullrich (2005), „Analyse von Tonhöhenordnungen. Allen Fortes pitch-classset-System ", in: Musiktheorie (= Handbuch der Systematischen Musikwissenschaft, Bd. 2), hg. von Helga de la Motte-Haber und Oliver Schwab-Felisch, Laaber: Laaber, 391-408.

Schleifer, Eliyahu (2001), »Ashkenazi« (= »ewish music« III.3), in: The New Grove Dictionary of Music and Musicians, 2. Auflage, hg. von Stanley Sadie, Bd. 13, London: Macmillan, 51-59.

Schmidt-Beste, Thomas (1997), »Ab ca. 1470« (= »Modus« III), in: Die Musik in Geschichte und Gegenwart. Allgemeine Enzyklopädie der Musik, hg. von Ludwig Finscher, 2. Auflage, Sachteil Bd. 6, Kassel: Bärenreiter / Stuttgart: Metzler, 417-431.

Schneider, Marius (1966), »Außereuropäisch« (= »Tonsysteme« C), in: Die Musik in Geschichte und Gegenwart. Allgemeine Enzyklopädie der Musik, 1. Auflage, hg. von Friedrich Blume, Bd. 13, Kassel: Bärenreiter, 547-558.

Schneider, Sigrun (1975), Mikrotöne in der Musik des 20. Jahrhunderts. Untersuchungen zu Theorie und Gestaltungsprinzipien moderner Kompositionen mit Mikrotönen, Bonn: Verlag für systematische Musikwissenschaft.

Schneider, Albrecht (1997), Tonhöhe Skala Klang. Akustische, tonometrische und psychoakustische Studien auf vergleichender Grundlage, Bonn: Verlag für Systematische Musikwissenschaft.

Schönberg, Arnold (1957), Die formbildenden Tendenzen der Harmonie, Mainz: Schott.

Schrader, Christiane (2017), „Warum ist die Mentalisierungsbasierte Therapie (MBT) für die Psychotherapie im Alter besonders interessant? «, Psychotherapie im Alter 14/1, 51-64.

Schultz-Venrath, Ulrich (2017), »Therapie von Persönlichkeitsstörungen aus Sicht der Mentalisierungsbasierten Therapien", Persönlichkeitsstörungen. Theorie und Therapie 21/1, 25-38.

Schuster-Craig, John (1990), »An Eighth Mode of Limited Transposition«, The Music Review 51/4, 296-306.

Schweikert, Uwe (2001), „Quattro pezzi sacri«, in: Verdi Handbuch, hg. von Anselm Gerhard und Uwe Schweikert, Metzler: Stuttgart / Kassel: Bärenreiter, 504-508.

Sisask, Urmas (2005), Gloria patri... 24 Hymns, Helsinki: Fennica Gehrman.

Sprenger, Sebastian (2009), „Über einige pentatonische Melodiebildungen in den Liedern Franz Schuberts", in: Musiktheorie an ihren Grenzen: Neue und Alte Musik. 3. Internationaler Kongress für Musiktheorie, 10.-12. Oktober 2003, Musik-Akademie der Stadt Basel, hg. von Angelika Moths, Markus Jans, John MacKeown und Balz Trümpy, Bern: Lang, 289-304.

Taruskin, Richard (1985), „Chernomor to Kashchei: Harmonic Sorcery; Or, Stravinsky's >Angle«", Journal of the American Musicological Society 38/1, 72-142.

Thompson, William F. (2013), »Intervals and Scales«, in: The Psychology of Music, hg. von Diana Deutsch, 3. Auflage, London: Academic Press, 107-140.

Thorau, Christian (2012), Vom Klang zur Metapher. Perspektiven der musikalischen Analyse, Hildesheim: Olms. 
Vetter, Walther (1956), »Antike Musik« (= "Griechenland«A), in: Die Musik in Geschichte und Gegenwart. Allgemeine Enzyklopädie der Musik, 1. Auflage, hg. von Friedrich Blume, Bd. 5, Kassel: Bärenreiter, 840-865.

Vieru Anatol (1993), The Book of Modes (2 Bde.), Bukarest: Editura Muzicală.

Wiering, Frans (2001), The Language of the Modes. Studies in the History of Polyphonic Modality, New York: Routledge.

Ziegler, Reinald (2009) »Das so genannte >dritte Tonartensystem und frühen 18. Jahrhunderts", in: Musiktheorie an ihren Grenzen: Neue und Alte Musik. 3. Internationaler Kongress für Musiktheorie, 10.-12. Oktober 2003, MusikAkademie der Stadt Basel, hg. von Angelika Moths, Markus Jans, John MacKeown und Balz Trümpy, Bern: Lang, 393-408. 